\title{
El síndrome de Heyde
}

\author{
Dr. Jorge Estigarribia Passaro
}

\section{Resumen}

Desde que Edward Heyde vislumbró en 1958 una misteriosa asociación entre estenosis aórtica y hemorragia digestiva han transcurrido seis décadas y se ha suscitado no poca controversia. En la época en que fue propuesta, el estatus técnico y metodológico de la ciencia médica y una interpretación sesgada de su idea original impidieron obtener un sustento estadístico y fisiopatológico que le otorgara un amplio reconocimiento como entidad clínica individual.

Los avances en varias disciplinas permitieron demostrar que su frecuencia de presentación excede el efecto del azar, además de esclarecer con precisión y elegancia sus mecanismos fisiopatológicos. Su consolidación como síndrome nos revela una verdadera encrucijada entre la cardiología, la gastroenterología, la hematología y el laboratorio, especialidades involucradas tanto en su proceso de comprensión como en su manejo práctico en la actualidad. Sin embargo, a pesar de tener una incidencia no desdeñable y adquirir un papel central en la conducción clínica de la estenosis aórtica, esta entidad parece haber pasado de ser resistida a relativamente ignorada.

Con el objetivo de contribuir a su visibilidad, la presente revisión ofrece un panorama integral sobre el tema, incluyendo una perspectiva histórica de los principales aportes en pos de su conocimiento y un abordaje en profundidad de sus mecanismos, las claves de su detección clínica y su impacto en el manejo de la estenosis aórtica y otras entidades con fisiopatología afín.

Palabras clave: SÍNDROME DE HEYDE

REVISIÓN

FISIOPATOLOGÍA

DIAGNÓSTICO

MANEJO

\section{Heyde syndrome}

\section{Summary}

Since Edward Heyde perceived in 1958 a mysterious association between aortic stenosis and gastrointestinal bleeding, six decades have passed and no little controversy has arisen.

At the time it was proposed, the technical and methodological status of medical science and a biased interpretation of his original idea prevented obtaining a statistical and pathophysiological support that would grant it wide recognition as and individual clinical entity.

Advances in several disciplines allowed to demonstrate that its frequency of presentation exceeds the effect of chance, besides clarifying with precision and elegance its pathophysiological mechanisms. Its consolidation as a syndrome reveals a true crossroads between Cardiology, Gastroenterology, Hematology and Laboratory, specialties involved both in its understanding process and in its practical management today.

However, despite having a not negligible incidence and acquiring a central role in the clinical conduction of aortic stenosis, this entity seems to have gone from being resisted to relatively ignored.

With the objective of contributing to its visibility, this review offers a comprehensive overview of the subject, covering the main historical contributions to its knowledge and approaching in depth its mechanisms, the keys to its clinical detection and its impact on the management of aortic stenosis and other entities with related pathophysiology. Key words: $\quad$ HEYDE'S SYNDROME

REVIEW

PATHOPHYSIOLOGY

DIAGNOSIS

MANAGEMENT

Departamento de Cirugía. Instituto Nacional de Cirugía Cardíaca.

Luis A. de Herrera 2275. Montevideo, Uruguay.

Correo electrónico: jorgeestigarribia@movinet.com.uy

El autor declara no tener conflicto de intereses.

El presente artículo no tiene fuentes de financiación.

Recibido Set 30, 2021; aceptado Oct 18, 2021 


\section{Síndrome de Heyde}

\section{Resumo}

Desde que Edward Heyde imaginou em 1958 uma misteriosa associação entre estenose aórtica e hemorragia gastrointestinal, seis décadas se passaram e não houve pouca controvérsia. Na época em que foi levantada, o estado técnico e metodológico da ciência médica e uma interpretação tendenciosa de sua ideia original impediram a construção de um apoio estatístico e fisiopatológico que lhe concederia amplo reconhecimento como entidade clínica individual. Avanços em diversas disciplinas permitiram demonstrar que sua frequência de apresentação excede o efeito do acaso, além de esclarecer com precisão e elegância seus mecanismos fisiopatológicos. Sua consolidação como síndrome revela uma verdadeira encruzilhada entre Cardiologia, Gastroenterologia, Hematologia e Laboratório, especialidades envolvidas tanto em seu processo de compreensão quanto em sua gestão prática hoje.

No entanto, apesar de ter uma incidência não desprezível e adquirir um papel central no manejo clínico da estenose aórtica, esta entidade parece ter passado de resistida para relativamente ignorada.

Com o intuto de contribuir para sua visibilidade, esta revisão oferece um panorama abrangente do tema, revendo as principais contribuições históricas ao seu conhecimento e abordando em profundidade seus mecanismos, as chaves para sua deteç̧ão clínica e seu impacto na gestão da estenose aórtica e outras entidades com fisiopatologia relacionada. Palavras chave: SÍNDROME DE HEYDE

REVISÃO

FISIOPATOLOGIA

DIAGNÓSTICO

GESTÃO

"Investigar es ver lo que todo el mundo ha visto, $y$ pensar lo que nadie más ha pensado". Albert SzentGyörgyi. Médico e investigador húngaro. Premio Nobel de Medicina en 1937.

"La ciencia se compone de errores, que a su vez son los pasos hacia la verdad".

Julio Verne. Escritor visionario, poeta y dramaturgo francés (1828-1905).

\section{Introducción}

Durante mucho tiempo la estenosis aórtica fue interpretada — quizás con una mirada excesivamente mecanicista - como una patología sencilla y de comprensión lineal. Básicamente, se trataba de una enfermedad de causa degenerativa, congénita o secuelar reumática, de diagnóstico clínico relativamente simple, caracterizada por una obstrucción progresiva del tracto de salida del ventrículo izquierdo y una historia natural adversa, cuya única solución en su etapa sintomática era el levantamiento del obstáculo mediante el reemplazo valvular quirúrgico.

En las últimas dos décadas, nueva información sobre su patogenia y factores de riesgo, la caracterización de diferentes e intrincados cuadros hemodinámicos, cambios sustanciales en las directivas de profilaxis de la endocarditis infecciosa, la definición de nuevos marcadores pronósticos en el paciente asintomático y el advenimiento y consolidación del implante valvular percutáneo aumentaron notablemente la complejidad del manejo de la enfermedad. Esto obligó, a menudo, a que el equipo terapéutico tomara difíciles decisiones en cada etapa del proceso asistencial, al punto de haber consagrado la necesidad de un heart team.

Un aspecto señalado no tan recientemente, aunque muy poco reconocido y en ocasiones negado, es su asociación sindromática con sangrado digestivo, intercurrencia que complica su evolución e impone un particular desafío al momento proveer una solución definitiva a la enfermedad.

En esta revisión se tratan aspectos históricos en el conocimiento del síndrome, profundizando en su particular fisiopatología y en la discusión sobre su propia existencia, y se analizan los problemas de diagnóstico que plantea, sus alternativas de tratamiento y la importancia de su reconocimiento en el manejo correcto de la valvulopatía.

Acerca del epónimo y la definición del síndrome La primera noción sobre esta asociación surgió de un intercambio epistolar.

En julio de 1958 el Dr. Edward Heyde, internista en Vancouver, Washington, en una carta al editor del New England Journal of Medicine, informa su experiencia con 10 pacientes de edad avanzada con claro diagnóstico clínico de estenosis aórtica severa que sufrieron sangrado digestivo grave cuyo origen exacto no pudo establecerse ${ }^{(1)}$ (figura 1). A su parecer, el cuadro hemorrágico se presentaba con una frecuencia mayor que entre individuos sin estenosis aórtica.

Esta observación fue rápidamente respondida con otras dos cartas en la misma publicación relatando experiencias coincidentes ${ }^{(2,3)}$, en las que 

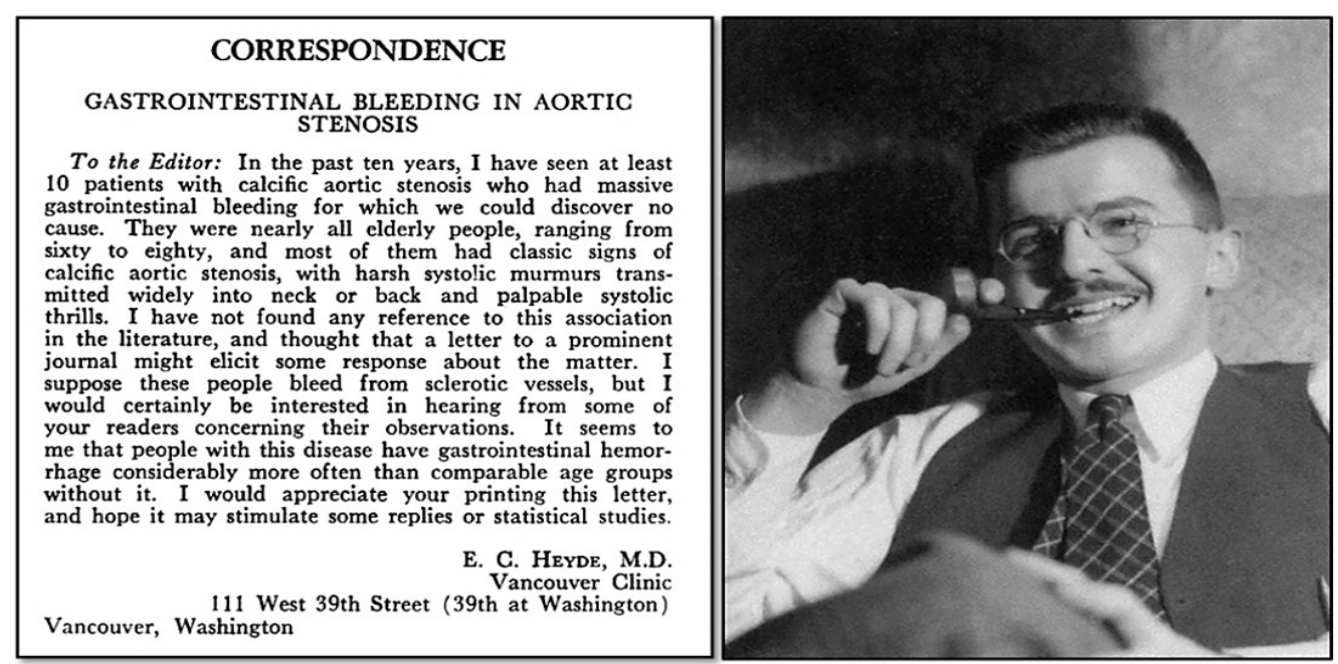

Figura 1. Izquierda: Reproducción de la carta en la que Edward Heyde sugirió por primera vez una asociación entre estenosis aórtica severa y hemorragia gastrointestinal ${ }^{(1)}$. Derecha: una imagen de Heyde en 1937.

se destaca de nuevo la edad avanzada de los pacientes y la recurrencia e incierto origen del sangrado digestivo, presentándose en una de ellas una apreciación estadística básica que apoyaría la asociación más allá del azar.

Desde entonces esta situación fue conocida como síndrome de Heyde, que en sentido amplio y respetando la publicación original del autor consiste en la asociación de estenosis aórtica con hemorragia digestiva severa, aunque en numerosos informes y revisiones subsiguientes se la ha restringido incorrectamente a la coexistencia de la valvulopatía con angiodisplasias gastrointestinales — de hecho, la lesión causal más frecuente-, con o sin sangrado manifiesto.

También se ha incluido en la definición, con mayor propiedad, a la coexistencia de una anemia ferropénica crónica de causa no identificada con una estenosis aórtica severa.

Debe notarse que la mayoría de los casos reportados en las mencionadas cartas correspondían a pacientes añosos, con sangrado a menudo recurrente y sin causa identificable — todo lo cual sugiere fuertemente angiodisplasias gastrointestinales- y que la asociación fue propuesta en base a datos clínicos en una época sin disponibilidad de ultrasonido cardíaco, endoscopía colónica ni procedimientos de sustitución valvular, los que, como veremos, representan la solución más apropiada para ambos componentes del cuadro.

En lo sucesivo se han publicado numerosos casos clínicos en distintas situaciones y con diferentes propuestas de tratamiento, así como múltiples revisiones. Sin embargo, su manejo no está aún protocolizado y las guías clínicas se refieren solo de manera colateral a esta asociación.

\section{¿Realidad o mito?}

Hubo un inicial escepticismo sobre una verdadera vinculación entre las dos entidades más allá del azar, interpretándose su coexistencia como un hallazgo casual, sin un nexo patogénico común o interdependiente.

Tal como sugerían las comunicaciones originales, los primeros estudios de asociación mostraron una significativa correlación entre estenosis aórtica y sangrado gastrointestinal de origen oscuro.

Este hecho y la publicación creciente de casos clínicos en los que se identificaron angiodisplasias como causa del sangrado condujeron a una definición más restringida del síndrome, y el objetivo de los subsiguientes estudios caso-control fue reflejo de este cambio. Quienes dudan de que el síndrome de Heyde sea una verdadera entidad ${ }^{(4)}$ argumen- $^{-}$ tan que tanto la estenosis aórtica como las angiodisplasias digestivas incrementan su prevalencia con la edad, de modo que su presencia simultánea en el adulto añoso es un hallazgo esperable. Sin embargo, un abordaje limitado a una mayor coexistencia de ambas lesiones resulta inapropiado, atendiendo a la definición original del síndrome, la dificultad en el diagnóstico de angiodisplasias - sobre todo sin sangrado activo-y a que la valvulopatía podría generar un mecanismo que condicione una tendencia al sangrado digestivo sin favorecer necesariamente su desarrollo, posibilidad ya planteada por Boley y Brandt en $1986^{(5)}$. 
En otras palabras, la consolidación del síndrome no depende de demostrar que las angiodisplasias son más prevalentes en pacientes con estenosis aórtica o viceversa, sino que estas u otras lesiones gastrointestinales sangran más a menudo en presencia de la valvulopatía que en su ausencia. La formulación de una explicación fisiopatológica consistente debería considerarse un argumento adicional en favor de su existencia, y la remisión de la tendencia hemorrágica con la resolución de la valvulopatía tendría valor confirmatorio.

Dada su complejidad, la caracterización del síndrome de Heyde fue una construcción gradual y colaborativa que recibió sucesivos aportes desde la investigación clínica, la cardiología, la gastroenterología, la hematología y el laboratorio especializado. A continuación trataremos de reconstruir este proceso.

\section{a) Estudios de asociación}

Williams en 1961 estudió en el Massachusetts General Hospital un grupo de 1.443 pacientes consecutivos con sangrado gastrointestinal ${ }^{(6)}$. Los 1.348 casos con causa demostrable no tuvieron una prevalencia significativamente mayor de estenosis aórtica $(5,0 \%)$ con respecto a controles pareados por edad y sexo (4,4\%). Sin embargo, el $25,5 \%$ de los 95 pacientes con sangrado de origen oscuro (la mayoría con edades entre la sexta y octava década) tenían estenosis aórtica, contra 5,2\% en los controles $(p<0,01)$.

McNamara y col. compararon en 1968 la frecuencia de sangrado digestivo entre pacientes intervenidos por valvulopatía aórtica versus mitral (en ambos casos estenosis, insuficiencia o doble lesión), detectando un $12 \%$ de episodios en los pacientes aórticos y un 4\% en mitrales $(p<0,05)$, incluyendo varios que ocurrieron en el posoperatorio bajo prescripción de anticoagulantes ${ }^{(7)}$. Los sangrados con causa detectada se debieron a ulcus duodenal o gastritis; en todos los pacientes mitrales pudo determinarse el origen, mientras que 19,2\% de los aórticos fueron idiopáticos.

Cody y col. ${ }^{(8)}$ encontraron, en una población de 275 pacientes con estenosis aórtica observados en la Clínica Mayo entre 1964 y 1969, una prevalencia de sangrado digestivo idiopático de $2,6 \%$ versus $0,025 \%$ en un grupo control $(p<0,001)$.

En el estudio de Shoenfeld y col. publicado en $1980^{(9)}$, de 152 pacientes intervenidos por estenosis aórtica, cuatro $(2,6 \%)$ presentaron sangrado gastrointestinal idiopático versus ninguno de 152 pacientes operados por estenosis mitral.

Asimismo, entre 154 pacientes ingresados por sangrado gastrointestinal, nueve $(5,8 \%)$ eran portadores de estenosis aórtica, mientras que en un grupo control de 154 pacientes emparejados por edad ingresados por otros motivos, solo tres (1,9\%) presentaron la valvulopatía.

En estos relevamientos iniciales ya se percibía una asociación particular entre estenosis aórtica y sangrado de causa no determinada, al menos con los métodos de estudio disponibles en la época.

En 1986 Greenstein y col. ${ }^{(10)}$ compararon la prevalencia de hemorragia gastrointestinal entre 1.811 pacientes con estenosis aórtica y 1.812 con estenosis mitral admitidos consecutivamente en el Mount Sinai Medical Center (Miami, Estados Unidos). Encontraron 21 sangrados entre los primeros y solo uno entre los segundos ( $<<0,001)$.

En 1988 Imperiale y col. efectuaron un análisis cuantitativo de los cuatro primeros estudios retrospectivos caso-control mencionados, señalando algunos sesgos al aplicarles un criterio metodológicamente exigente ${ }^{(11)}$. Realizaron un análisis de doble sentido, considerando tanto el sangrado gastrointestinal idiopático como "resultado" (en cuyo caso la estenosis aórtica es la "exposición") como la situación inversa. En el análisis de dos de los estudios encontraron "una fuerte asociación entre estenosis aórtica y sangrado gastrointestinal idiopático”. Sin embargo, la conclusión general fue que las imprecisiones metodológicas mencionadas impedían aceptar una evidencia fuerte a favor de la asociación de estenosis aórtica con sangrado idiopático o con angiodisplasias, a pesar de que los estudios analizados no hacen referencia a esta causa específica de sangrado.

Mehta y col. en $1989^{(12)}$ y Oneglia y col. en $1993^{(13)}$ revisaron respectivamente los registros de 29 y 59 pacientes portadores de angiodisplasias documentadas por endoscopía y evaluados con ecocardiograma Doppler. En el primer grupo, no se encontró estenosis aórtica severa en ningún paciente; en el segundo, solo en uno (1,6\%). Sin embargo, la hemorragia gastrointestinal no fue la variable considerada en la búsqueda de asociación con la valvulopatía, el número de pacientes fue pequeño y ambos carecían de grupo control.

Bhutani y col. ${ }^{(14)}$ en 1995 publicaron un estudio prospectivo no randomizado con 40 pacientes portadores de angiodisplasias y 37 controles emparejados por edad, sexo, indicación y tipo de examen endoscópico. En ninguno de los grupos se detectó ningún caso de estenosis aórtica, a pesar de que la esclerosis valvular resultó relativamente frecuente. Los autores, mediante una evaluación estadística y estableciendo varios subgrupos, concluyeron que la valvulopatía aórtica no juega un rol causal en las angiodisplasias o el sangra- 
do gastrointestinal. No obstante, dado el $n$ total tan pequeño y con solo 26 pacientes del grupo angiodisplasia y 23 controles presentando sangrado manifiesto u oculto, el bajo poder estadístico del estudio no permitió confirmar la hipótesis nula.

El estudio de Batur y col. publicado en $2003^{(15)}$ parece demostrar, en cambio, una asociación significativa entre angiodisplasias y estenosis aórtica, informando una prevalencia de $31,7 \%$ de estenosis aórtica de cualquier severidad en 63 pacientes con angiodisplasias diagnosticadas con endoscopía o arteriografía contra 14,0\% en 92.012 controles $(p<0,001)$. Considerando solo la estenosis aórtica moderada y severa, las cifras son de $15,9 \%$ y $6,0 \%$, respectivamente (p < 0,001). En comparación, la prevalencia de estenosis mitral de cualquier entidad no difirió significativamente entre la población con angiodisplasias y la población general (1,6\% versus $6,0 \%$ respectivamente; $\mathrm{p}=0,14)$ y tampoco la estenosis mitral significativa $(0,0 \%$ versus $1,9 \% ; p=0,27)$.

Pate y col. ${ }^{(16)}$ revisaron los registros de los pacientes externados de los hospitales públicos de Irlanda entre los años 1997 y 2001 (casi 3,8 millones) que al momento del alta tuvieran un diagnóstico (primario o secundario) de estenosis aórtica, presencia de sangrado gastrointestinal debido presuntamente a angiodisplasias, o ambos. El diagnóstico primario o secundario de sangrado gastrointestinal se registró en $0,2 \%$ de la población total, mientras que entre los pacientes con diagnóstico de estenosis aórtica se observó en $0,9 \%$ (p < 0,0001; OR 4,5; IC 95\% 3,06,8) (figura 2).

En suma, y aún reconociendo una serie de imperfecciones metodológicas y limitaciones diagnósticas propias de la época, los estudios que tuvieron como objetivo investigar una posible asociación entre estenosis aórtica y sangrado digestivo ya demostraban resultados coincidentemente positivos.

\section{b) Propuestas patogénicas}

En teoría, una asociación más allá del azar entre estenosis aórtica y sangrado digestivo podría deberse a tres posibles situaciones:

1) Que la estenosis aórtica coincida con alguna lesión digestiva potencialmente sangrante (por ejemplo, con angiodisplasias) con una frecuencia mayor a la esperable.

2) Que la estenosis aórtica favorezca de algún modo el sangrado de una lesión digestiva.

3) Una combinación de ambos mecanismos.

Desde el inicio, los esfuerzos para explicar el vínculo entre estenosis aórtica y sangrado digestivo se enfocaron en desentrañar el mecanismo por

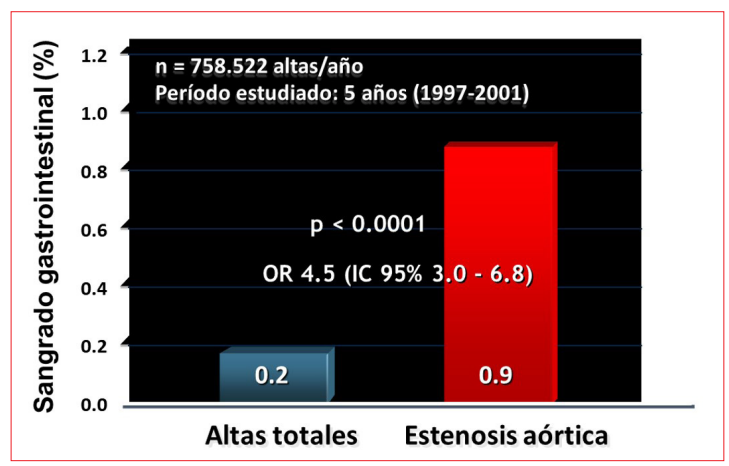

Figura 2. De un total de 3.792.609 altas hospitalarias, la prevalencia de sangrado gastrointestinal resultó significativamente mayor entre los pacientes con diagnóstico de estenosis aórtica que en la población global de pacientes externados. Representación gráfica elaborada de acuerdo con los datos publicados por Pate y colaboradores ${ }^{(16)}$.

el cual esta valvulopatía podría favorecer el desarrollo de angiodisplasias, que al igual que en los estudios caso-control concitaron la atención casi excluyente de los investigadores.

La primera sugerencia de una "malformación vascular" como causa del sangrado fue formulada por Cattell en 1965 —citado por Jacobson en uno de los casos del Massachusetts General Hospital $^{(17)}$ — quien planteó la hemicolectomía derecha a ciegas en presencia de estenosis aórtica y sangrado digestivo bajo recidivante sin causa detectable, en virtud de su experiencia con varios pacientes en los que la hemorragia provenía de lesiones vasculares del colon ascendente y cesaba luego de la intervención. Esta interpretación fue retomada por Boss y Rosenbaum en 1971, quienes además de citar dicha fuente presentan un caso fatal con hallazgo de ectasias de venas submucosas en la necropsia ${ }^{(18)}$.

La tendencia al sangrado recidivante del colon derecho en pacientes con estenosis aórtica continuó reportándose con frecuencia, al punto de que Shbeeb y col. plantearon el concepto de eje válvula aórtica-colon ${ }^{(19)}$, admitiendo la posible localización del sangrado en el resto del colon e incluso en el intestino delgado o en puntos múltiples.

Variados mecanismos fueron propuestos para explicar la patogénesis de las angiodisplasias. Algunos, sin vínculo directo con la estenosis aórtica, transitan desde el origen congénito o en una arteritis sifilítica hasta anormalidades del tejido conjuntivo. Se ha planteado con mayor fundamento una contracción incrementada y sostenida de la muscularis mucosae intestinal, generando ya sea una obstrucción intermitente de las venas submucosas, con congestión capilar, incompetencia de los esfínteres precapilares y desarrollo de colate- 


\begin{tabular}{|cl}
\hline \multicolumn{2}{|l}{$\begin{array}{l}\text { Tabla 1. Clasificación de la enfermedad de von } \\
\text { Willebrand. }\end{array}$} \\
\hline Tipo & Descripción \\
\hline 1 & Déficit cuantitativo parcial del fvW. \\
2 & Déficit cualitativo del fvW. \\
$2 \mathrm{~A}$ & $\begin{array}{l}\text { Disminución de la adhesión plaquetaria } \\
\text { dependiente del fvW con deficiencia } \\
\text { selectiva de los multímeros de alto peso } \\
\text { molecular. }\end{array}$ \\
\hline $2 \mathrm{~B}$ & $\begin{array}{l}\text { Aumento de la afinidad por la proteína } \\
\text { 1b plaquetaria. }\end{array}$ \\
\hline $2 \mathrm{M}$ & $\begin{array}{l}\text { Disminución de la adhesión plaquetaria } \\
\text { dependiente del fvW sin deficiencia } \\
\text { selectiva de los multímeros de alto peso } \\
\text { molecular. }\end{array}$ \\
\hline $2 \mathrm{~N}$ & $\begin{array}{l}\text { Afinidad por unión al factor VIII } \\
\text { marcadamente disminuida. }\end{array}$ \\
\hline 3 & \begin{tabular}{l} 
Déficit virtualmente completo del fvW. \\
\hline
\end{tabular}
\end{tabular}

Guía del National Heart, Lung and Blood Institute (NHLBI) USA, 2007 (67). fvW: factor von Willebrand.

rales arteriovenosas, o hipoxia tisular crónica que induce vasodilatación mediada por vía simpática y/o angiogénesis a través de mediadores químicos como el factor de crecimiento vascular endotelial (VEGF).

Por analogía, se ha planteado que en la estenosis aórtica un bajo volumen minuto con hipoperfusión e hipoxia crónica de la mucosa intestinal podría favorecer tanto el desarrollo de angiodisplasias por estímulo de la angiogénesis como su tendencia al sangrado ${ }^{(20)}$.

En 1986, Gill y col. ${ }^{(21)}$ demostraron una alteración de la hemostasis como causa de sangrado no digestivo en afecciones cardiovasculares caracterizadas por gradientes de presión elevados. Estudiaron de manera minuciosa doce pacientes con cardiopatías congénitas no cianóticas e historia de sangrados mucocutáneos (sugestivos de enfermedad de von Willebrand) y/o alteraciones en la hemostasis básica preoperatoria.

Solo en la mitad de sus casos encontraron una baja concentración del factor von Willebrand (fvW), pero en la mayoría demostraron un descenso de su actividad biológica y en todos ellos un déficit selectivo de sus multímeros de alto peso molecular con electroforesis en gel de agarosa, planteando un mecanismo de adsorción a nivel plaquetario a causa de la turbulencia o una alteración enzimática alternativa.

En cinco pacientes reestudiados luego de la corrección, excepto en uno en el que el gradiente se mantuvo (a nivel de un banding pulmonar), se normalizó el patrón multimérico. Los pacientes no tenían historia familiar de sangrados, y en ningún caso el estudio de la familia mostró anomalías de la hemostasis, por lo cual interpretaron la condición como una forma adquirida de enfermedad de von Willebrand.

Warkentin y col. en $1992^{(22)}$ retoman este mecanismo fisiopatológico y lo aplican a la estenosis aórtica del adulto, proponiendo que el sangrado en el síndrome de Heyde, casi siempre originado en angiodisplasias, se debe a una enfermedad de von Willebrand adquirida de tipo $2 \mathrm{~A}$ (tabla 1 ). Interpretan que la pérdida de los grandes multímeros del fvW se produce por un incremento de la interacción entre fvW-plaquetas bajo las intensas fuerzas de cizalla (shear stress) a nivel de la lesión valvular (figura 3), y que el deterioro de la hemostasis resulta particularmente importante en lechos vasculares dilatados y tortuosos, donde la participación de los grandes multímeros del fvW es esencial.

En 1994, Tsai y col. ${ }^{(23)}$ demuestran en el laboratorio que la distribución de los multímeros de diferente peso molecular (PM) del fvW en plasma es determinada por una actividad enzimática proteolítica que se incrementa en relación directa con las fuerzas de cizalla del lecho vascular, a su vez dependientes de la velocidad del flujo. Ven en este mecanismo un regulador fisiológico basado en cambios en la conformación del sustrato que además estaría implicado en la caída de los multímeros de alto PM en ciertos desórdenes adquiridos del fvW, aunque no lo asocia expresamente al síndrome de Heyde.

En el año 2000, Pareti y col. ${ }^{(24)}$ estudian 22 pacientes portadores de estenosis aórtica antes y después de la sustitución valvular mediante agregometría y electroforesis del fvW, y concluyen que el déficit selectivo de los grandes multímeros, reversible con la corrección de la lesión, se debe a una proteólisis aumentada, probablemente por un cambio conformacional de la molécula con mayor exposición de la unión peptídica sensible a la enzima, aunque sin identificarla. Hasta entonces se sabía que algunas enzimas proteolíticas como la plasmina, la catepsina G y la calpaína eran capaces de fragmentar la molécula del fvW, pero en 2001 Fujikawa y col. logran purificar y secuenciar la enzima específica que regula fisiológicamente la composición multimérica del fvW mediante la escisión de la molécula a nivel de la unión Tir $1605 \mathrm{Met} 1606^{(25)}$. No posee activadores ni inhibidores naturales, y su regulación se realiza a nivel del sustrato por acción de las fuerzas de 


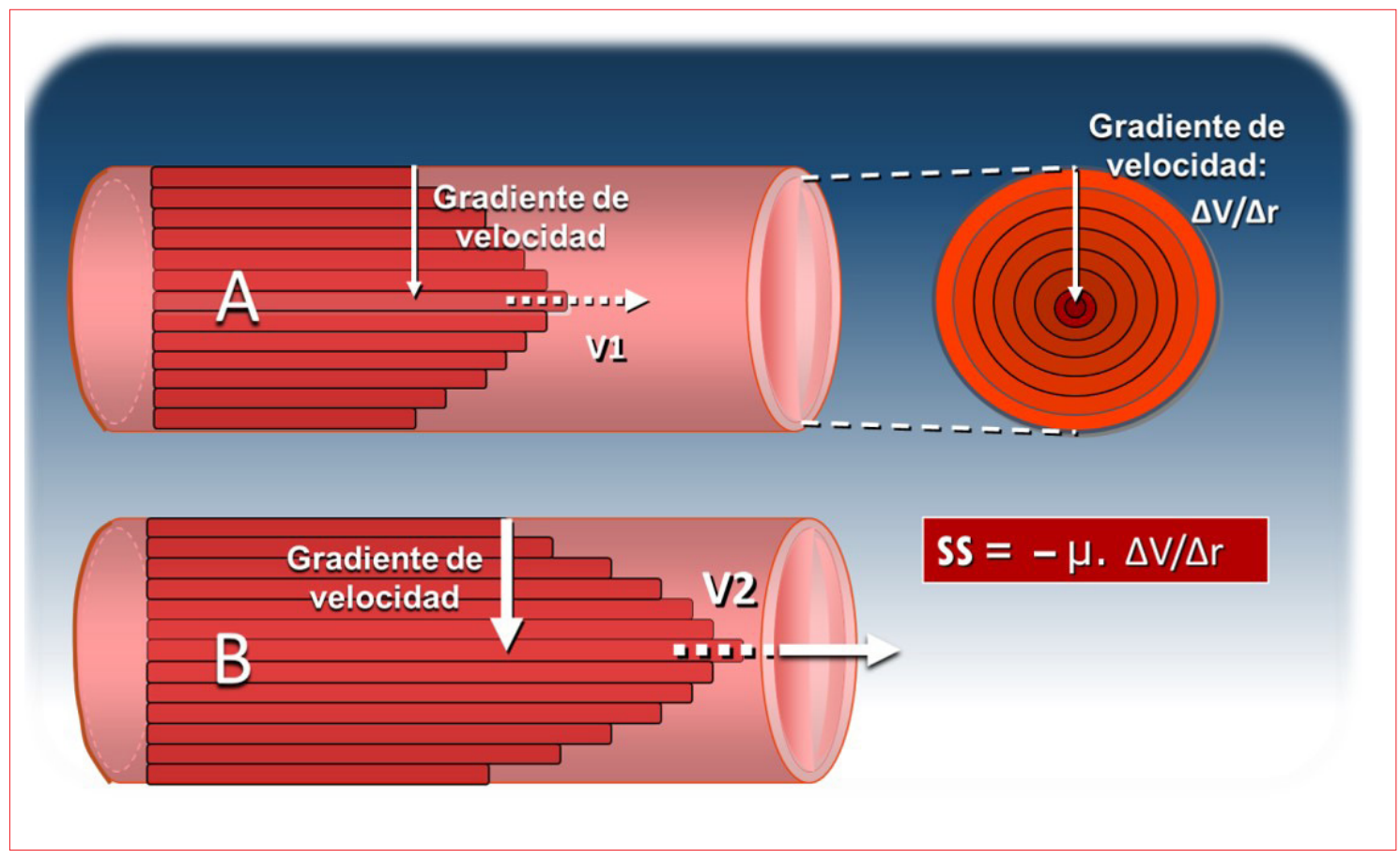

Figura 3. El concepto de shear stress, fuerza de cizallamiento, de fricción o tensión tangencial. A los efectos prácticos, la sangre se comporta en el sistema vascular como si fluyera en capas contiguas dispuestas en círculos concéntricos, con la máxima velocidad en el centro y la mínima en la capa externa adyacente a la pared. Esto genera un gradiente de velocidad cuya magnitud está dada por el cociente entre la diferencia de velocidades capa externa/ capa central y el radio del vaso $(\boldsymbol{\Delta V} / \mathbf{\Delta} \mathbf{r})$. El shear stress (SS) es el producto de este gradiente por el coeficiente de viscosidad dinámica $(\boldsymbol{\mu})$. Por lo tanto, cuando aumenta la velocidad circulatoria $(\mathbf{V} 1 \rightarrow \mathbf{V} 2)$ aumentan proporcionalmente el gradiente de velocidad y el shear stress, como sucede en B con respecto a A. Tanto la estenosis aórtica como las angiodisplasias generan altas velocidades circulatorias locales y son, por lo tanto, ámbitos de alto shear stress circulatorio.

cizalla en los lechos vasculares, que modifican la estructura terciaria del fvW y aumentan la exposición de la unión peptídica sensible al sitio activo de la enzima. Fue identificada como una metaloproteinasa perteneciente a la familia ADAMTS, designada como ADAMTS13.

En 2003, Vincentelli y col. ${ }^{(26)}$ encuentran una prevalencia elevada del síndrome entre pacientes coordinados para sustitución valvular $(21,4 \%)$ y demuestran una relación directa entre la severidad de la estenosis evaluada por gradiente transaórtico medio y el deterioro de los multímeros de alto PM del fvW. Confirman, además, la reversibilidad precoz del defecto hemostático luego de la cirugía y su recurrencia en pacientes con mismatch protésico, datos cruciales para establecer una relación de causalidad.

La primera guía clínica que reconoce la existencia del síndrome con una breve referencia a su fisiopatología - aunque sin mencionar el epónimo- es la actualización 2008 de la Guía de Manejo de Valvulopatías de 2006 del American College of Cardiology y la American Heart Association ${ }^{(27)}$. Sin embargo, en el documento no se establece su importancia clínica ni de definen pautas de conducta en esta situación.

En la tabla 2 se ordenan de manera cronológica las principales contribuciones en el conocimiento de la patogenia del síndrome de Heyde.

Dado que el fvW juega un papel central en el mecanismo íntimo de esta entidad, parece oportuno detenerse en su estructura y sus interacciones moleculares fundamentales ${ }^{(28-30)}$. Clásicamente se reconoce al fvW como una macromolécula con función esencial en la hemostasis, codificada por un gen situado en el brazo corto del cromosoma 12. Se compone de subunidades de 2.050 residuos de aminoácidos, cuyo precursor es sintetizado por las células endoteliales y los megacariocitos como una gran proteína que sufre varias modificaciones postraducción, la última es la formación de multímeros.

El fvW maduro es almacenado en las células endoteliales en los cuerpos de Weibel-Palade y liberado lentamente a la sangre circulante, donde existe una mezcla de multímeros de diferentes tamaños unidos por puentes disulfuro, desde dímeros de 500.000 daltons hasta formas altamente 


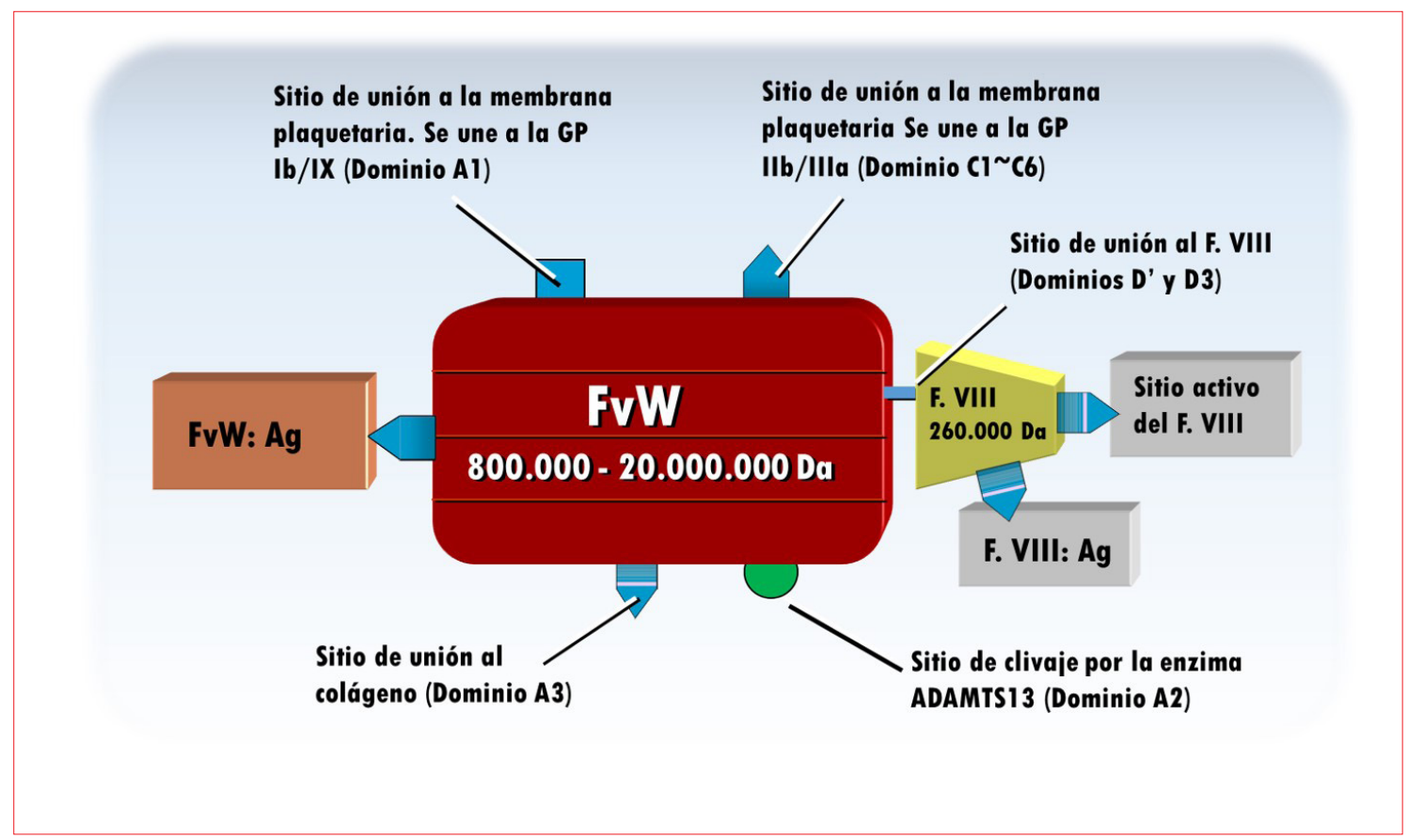

Figura 4. Representación esquemática de la estructura y dominios del factor von Willebrand (fvW). La molécula tiene un dominio responsable de la respuesta antigénica ( $\mathrm{fvW}: \mathrm{Ag}$ ), base de su dosificación en plasma, y otro para la unión y el transporte del factor VIII de la coagulación (F. VIII). En este se diferencian una secuencia antigénica (F. VIII: Ag) y un sitio activo responsable de su función biológica. Además, el fvW presenta un sitio de unión a los receptores del colágeno, otro para la glucoproteína Ib y otro para la glucoproteína IIb/IIIa de la membrana plaquetaria, así como un dominio donde se produce su clivaje por su regulador fisiológico, una metaloproteinasa específica, llamada ADAMTS13.

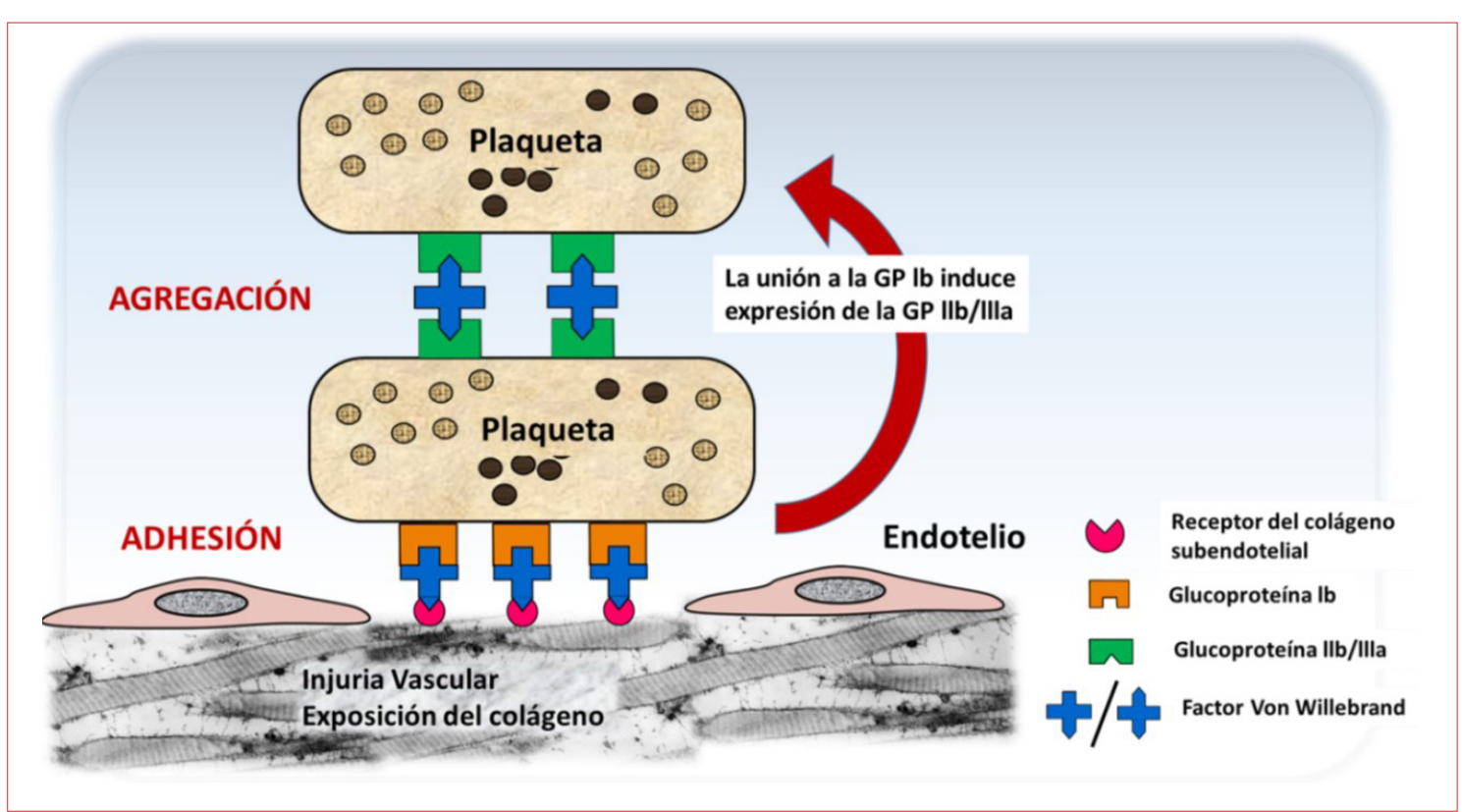

Figura 5. Papel del factor von Willebrand (fvW) en la adhesión y agregación plaquetarias. La lesión vascular expone los receptores de las fibras colágenas del subendotelio al flujo sanguíneo. Moléculas del fvW circulante actúan como puente entre estos receptores y la glucoproteína lb de la membrana plaquetaria mediante uniones específicas, iniciando el fenómeno de adhesión, el que, a su vez, desencadena una mayor expresión de la glucoproteína llb/llla de superficie y liberación de tromboxano A2 y ADP. Otras moléculas de fvW ofician de nexo entre plaquetas vecinas uniéndose a la glucoproteína IIb/IIIa, también a través de dominios moleculares especiales, dando lugar a la primera etapa de la agregación plaquetaria. Para que estos procesos sean eficientes, resulta esencial la conservación de los polímeros de alto peso molecular del fvW, principalmente en vasos con elevado shear stress, como las angiodisplasias. 
Tabla 2. Principales aportes en la caracterización clínica y patogénica del síndrome de Heyde.

1958 Informe original de Edward Heyde mediante una carta publicada en NEJM: 10 pacientes añosos con estenosis aórtica calcificada severa y sangrado intestinal masivo sin causa aparente ${ }^{(1)}$.

1965 Cattell (citado por Castleman y McNeely) sugiere una malformación vascular como causa del sangrado y aconseja la hemicolectomía derecha a ciegas para tratar hemorragias graves recidivantes de origen no definido ${ }^{(17)}$.

1986 Gill informa sobre una diátesis hemorrágica basada en un déficit de los multímeros de alto PM del fvW en cardiopatías congénitas no cianóticas con elevados gradientes, reversible con la corrección efectiva del defecto, interpretándola como una probable forma adquirida de la enfermedad de von Willebrand. Postula dos mecanismos posibles: la adsorción del fvW a las plaquetas o su degradación por un mecanismo enzimático ${ }^{(21)}$.

1987 King y col. reportan que casi todos los pacientes con síndrome de Heyde tratados con exploración abdominal sufren recurrencias de sangrado, mientras que $93 \%$ de los que reciben remplazo valvular aórtico se $\operatorname{curan}^{(38)}$.

1992 Warkentin retoma el mecanismo propuesto por Gill y lo enmarca en la estenosis aórtica del adulto, planteando que el síndrome se origina en una enfermedad de von Willebrand adquirida de tipo 2A "por incremento de la interacción plaquetas/fvW a nivel de la válvula estenótica", y sugiere un posible tratamiento de sostén hemostático ${ }^{(22)}$.

1994 Tsai y col. demuestran que la composición multimérica del fvW en sangre circulante es regulada por una actividad proteolítica modulada por las fuerzas de cizalla (shear stress) en los lechos vasculares a través de un cambio conformacional, y puede estar involucrada en la pérdida de los grandes multímeros en algunos desórdenes adquiridos del fvW $\mathrm{W}^{(23)}$.

2000 Pareti y col. proponen que la diátesis hemorrágica observable específicamente en la estenosis aórtica se sustenta en el mecanismo enzimático postulado por Tsai y col. ${ }^{(24)}$.

2001 Veyradier y col. encuentran déficit de los multímeros de alto PM y estenosis aórtica solo en las angiodisplasias sangrantes, no así en las no sangrantes ni en enterorragias por colopatía diverticular ${ }^{(68)}$. Fujikawa y col. purifican y secuencian la enzima específica responsable de la proteólisis del fvW, y la caracterizan como una metaloproteinasa perteneciente a la familia ADAMTS, denominada ADAMTS13 ${ }^{(25)}$

2002 Warkentin plantea que la elevada tensión de cizalla a nivel de la lesión aórtica puede incrementar la actividad de la metaloproteinasa específica recientemente identificada, con pérdida selectiva de los multímeros de alto PM del fvW. Reporta dos pacientes con recuperación laboratorial y cese persistente del sangrado a 10 años de la sustitución valvular ${ }^{(69)}$.

2003 Vicentelli y col. demuestran una relación inversa entre el gradiente transaórtico y la concentración del fvW. Reportan la remisión del sangrado luego de sustitución valvular quirúrgica, con recuperación funcional del fvW en estudios de función plaquetaria e inmunoelectroforesis y recidiva del cuadro en caso de mismatch prótesis-paciente ${ }^{(26)}$.

2006 Yoshida y col. reportan reducción del shear stress, mejoría de la agregabildad plaquetaria y recuperación de los multímeros de alto PM solo en los pacientes sin mismatch protésico ${ }^{(70)}$. La actualización de 2008 de la Guía de valvulopatías ACC/AHA 2006 reconoce la asociación de estenosis aórtica con un SvWA y sangrado en $20 \%$ de los pacientes, y su resolución con el reemplazo valvular ${ }^{(27)}$.

2012 Thompson reporta curación del síndrome con reemplazo valvular aórtico en $79 \%$ de los pacientes ${ }^{(39)}$.

PM: peso molecular; fvW: factor von Willebrand; ACC: American College of Cardiology; AHA: American Heart Association; SvWA: síndrome de von Willebrand adquirido.

polimerizadas de hasta 20.000 .000 daltons. Su estructura y sitios específicos de interacción biológica (dominios) se esquematizan en la figura 4.

El fvW cumple un papel central en la adhesión y la agregación de las plaquetas, actuando como un sensor de daño endotelial, formando un puente entre glucoproteínas específicas de la superficie plaquetaria y el subendotelio expuesto y entre receptores de plaquetas vecinas (figura 5). También tiene una participación importante en la coagulación por su unión y estabilización del factor VIII, al cual protege de la proteólisis y transporta hasta el sitio de injuria. La proporción en la que circulan sus multímeros es relevante para una hemos- tasis normal, dado que los de mayor PM tienen mayor actividad proagregante que los polímeros menores, y resultan críticos en lechos vasculares tortuosos y de alto flujo y, por lo tanto, con mayor shear stress, como las angiodisplasias.

Como contrapartida, un exceso de estos macropolímeros en plasma podría generar una adhesión y agregación plaquetarias descontroladas, lo que es evitado gracias a una sutil regulación por la enzima específica ADAMTS13, que los degrada en moléculas más pequeñas y de menor reactividad espontánea con las plaquetas. 
Tabla 3. El laboratorio en la enfermedad de von Willebrand adquirida de tipo $2 a$.

\section{Crasis sanguínea:}

Recuento plaquetario normal. Tiempo de sangría inconstantemente prolongado.

Dosificación del antígeno del factor von Willebrand (vWF: Ag). Mide su concentración plasmática:

Variable; puede ser normal o levemente disminuido (30-200 UI/100 ml).

Actividad del cofactor ristocetina del factor von Willebrand (vWF: RCo).

Mide su actividad biológica:

Generalmente disminuido $(<30 \mathrm{UI} / 100 \mathrm{ml})$.

Relación actividad del cofactor ristocetina/antígeno von Willebrand (vWF: RCo/vWF: Ag):

Generalmente disminuido (<0,5-0,7 [5070\%]).

Aglutinación plaquetaria inducida por ristocetina (RIPA):

Disminuida con dosis estándar de ristocetina; ausente con dosis reducida (LD-RIPA).

Actividad coagulante del factor VIII (F VIII: C):

Normal o levemente disminuida.

Tiempo de oclusión con el PFA-100 en condiciones de elevado shear stress:

Típicamente prolongado (> $300 \mathrm{~s}$ ).

Análisis de los multímeros del factor vW por electroforesis en gel de agarosa (inmunoblotting):

Estudio decisivo. Reducción o ausencia selectiva de los multímeros de alto peso molecular.

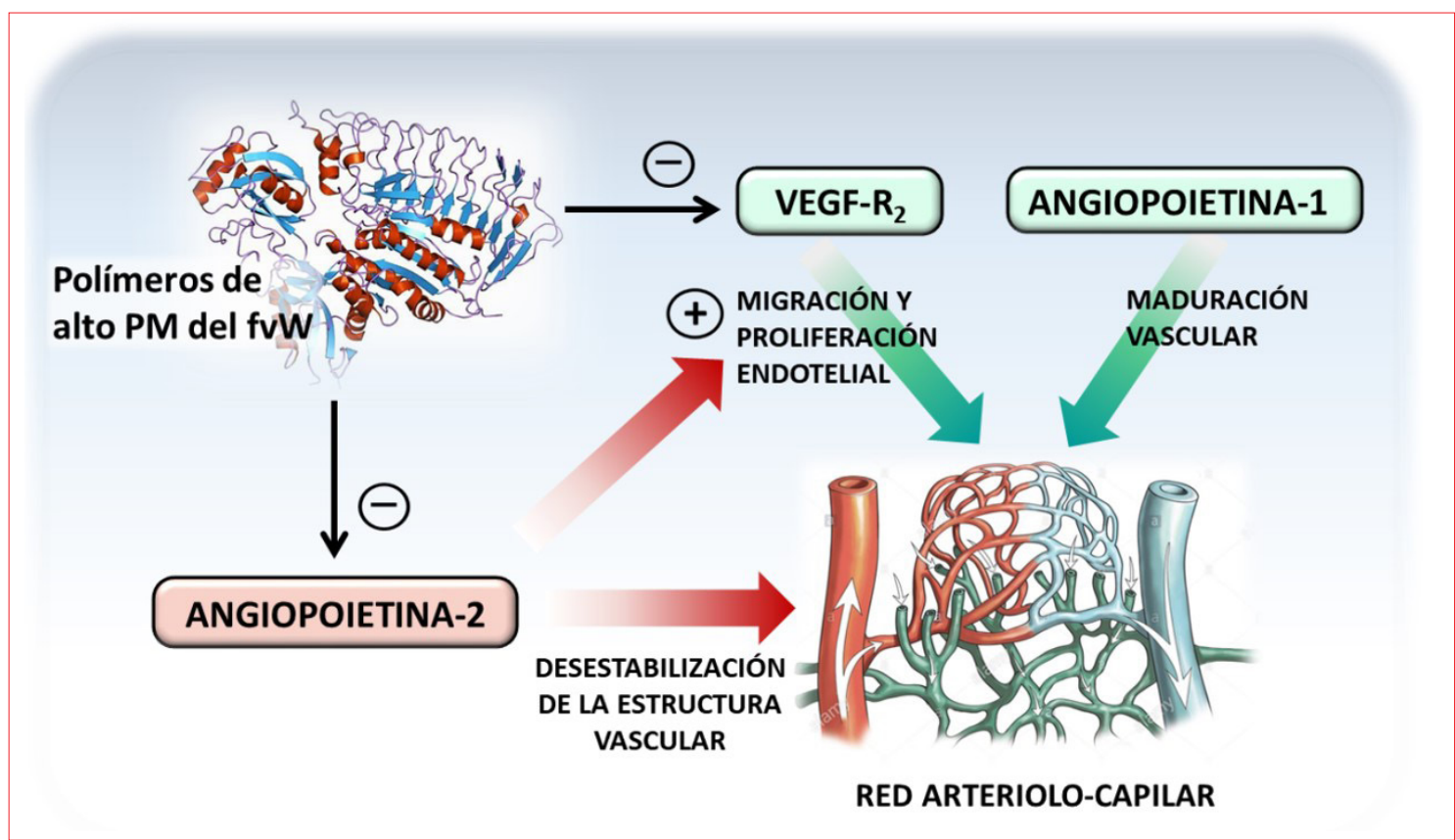

Figura 6. Rol del déficit del factor von Willebrand (fvW) en la génesis de angiodisplasias. El desarrollo de un sistema vascular normal depende, entre otros, del factor de crecimiento endotelial vascular (VEGF), que estimula la migración y la proliferación endotelial, y de la angiopoietina1, que favorece su maduración y estabilidad por reclutamiento de células musculares lisas y pericitos. La angiopoietina2 promueve angiogénesis en forma sinérgica con el VEGF, pero en exceso desestabiliza la estructura vascular, generando vasos displásicos. El fvW, y particularmente sus componentes de mayor peso molecular, ejercen un reconocido efecto antiangiogénico, por reducción de la expresión del receptor 2 del VEGF (VEGFR2) y control de la liberación de la angiopoietina2 de la célula endotelial. Su déficit determina, en consecuencia, un crecimiento excesivo y desordenado de lechos vasculares inmaduros, con desarrollo de angiodisplasias, según datos de Starke y col. ${ }^{(32)}$.

Nuevos roles del factor von Willebrand

Información reciente revela al fvW como una molécula de gran versatilidad funcional, que transporta y/o interactúa con más de 20 proteínas (bus molecular), y juega un rol activo, más allá de la hemostasis, en varios procesos que incluyen la inflamación, la proliferación de células de músculo liso, el desarrollo de metástasis tumorales y la angiogénesis, de la que se comporta como inhibidor $^{(31)}$. En efecto, ha sido frecuentemente reconocida la presencia de angiodisplasias en pacientes portadores de enfermedad de von Willebrand. 


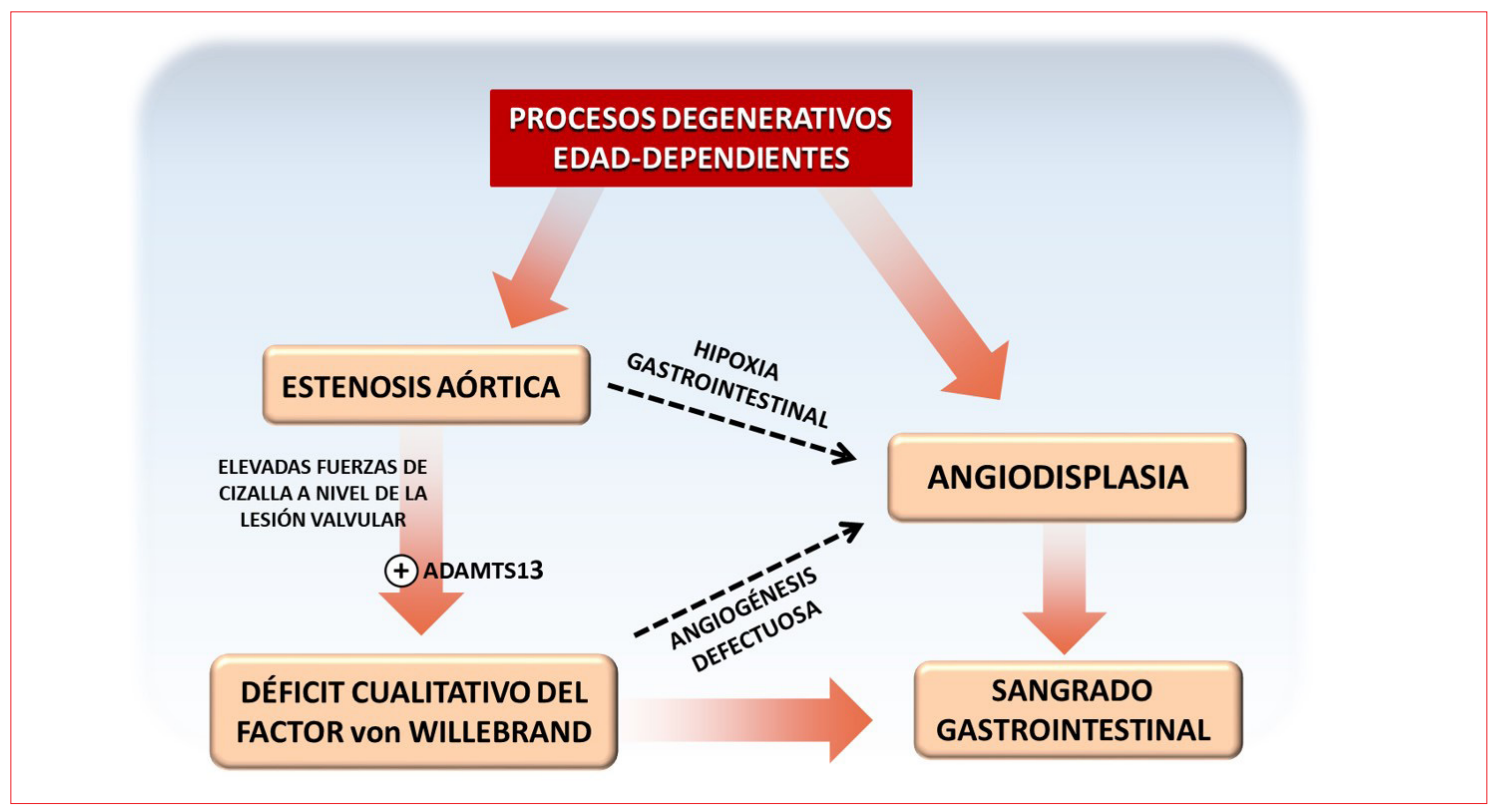

Figura 7. Concepto fisiopatológico unificador del síndrome de Heyde. Tanto la estenosis aórtica como las angiodisplasias aumentan su prevalencia con la edad, por procesos interpretados como "degenerativos". Existe incontrovertible evidencia de que la valvulopatía origina con frecuencia una diátesis hemorrágica por déficit selectivo de los multímeros de alto peso molecular del factor von Willebrand, originado en un exceso de actividad de su enzima proteolítica específica, la ADAMTS13. Esta deficiencia, definida como una enfermedad de von Willebrand adquirida de tipo $2 \mathrm{~A}$, favorece el sangrado de angiodisplasias coexistentes. Adicionalmente, se han dilucidado mecanismos moleculares por los cuales la estenosis aórtica puede inducir no solo el sangrado, sino el desarrollo de estas malformaciones vasculares (modificado de Sucker y col. ${ }^{(37)}$ ).

Nuevos estudios detallan mecanismos moleculares que vinculan el déficit de este factor al desarrollo de angiodisplasias, donde intervienen cambios en la actividad del VEGF y de las angiopoietinas 1 y 2 , entre otros factores no identificados con claridad $^{(22,33)}$ (figura 6).

El papel de la estenosis aórtica en la génesis de angiodisplasias encuentra apoyo adicional en el estudio ya mencionado de Batur y col. ${ }^{(15)}$ y en reportes con documentación endoscópica de desaparición total de angiodisplasias tras la resolución de la valvulopatía ${ }^{(34,35)}$.

Desde el punto de vista estructural, las angiodisplasias son ramilletes de capilares arteriales o venosos dilatados y tortuosos, de hasta $5 \mathrm{~mm}$ de diámetro, localizados en la submucosa del tracto gastrointestinal. Carecen de capa media y exhiben una permeabilidad aumentada con particular tendencia al sangrado, a menudo recurrente. Clásicamente, su origen se ha atribuido a cambios degenerativos, y su localización predominante se ha situado en el ciego y colon ascendente, pudiendo presentarse desde el cardias hasta el esfínter anal. El advenimiento de la videocápsula endoscópica permitió conocer su elevada frecuencia en el intestino delgado, un sector mayoritariamente ciego a los estudios endoscópicos convencionales, lo que, junto a su naturaleza de lesión plana y su localización frecuentemente multifocal, puede convertir la identificación del punto de un sangrado en un verdadero desafío diagnóstico ${ }^{(20,36)}$.

Considerando el amplio cuerpo de información disponible a la fecha, es posible formular con Sucker ${ }^{(37)}$ un cuadro fisiopatológico unificador que sustenta por doble vía la observación original de Heyde, apoyada por múltiples comunicaciones posteriores (figura 7).

\section{c) Regresión del defecto hemostático y del san- grado con la resolución de la valvulopatía}

Son numerosas las comunicaciones de casos individuales y de series de casos que, como prueba definitiva de la validez del síndrome, documentan la desaparición de la diátesis hemorrágica luego de la sustitución valvular aórtica, tanto en sus manifestaciones clínicas como en sus correlatos de laboratorio. Sea mediante sustitución valvular quirúrgica o implante percutáneo, el alivio del gradiente transvalvular y el aumento del área aórtica efectiva se acompañan de un cese duradero de los sangrados y la recuperación de los multímeros de alto PM del fvW.

De la serie de 91 pacientes con estenosis aórtica y sangrado gastrointestinal inexplicado de King y col. ${ }^{(38)}$ solo el $5 \%$ de los que se sometieron a exploración abdominal y resección intestinal ob- 


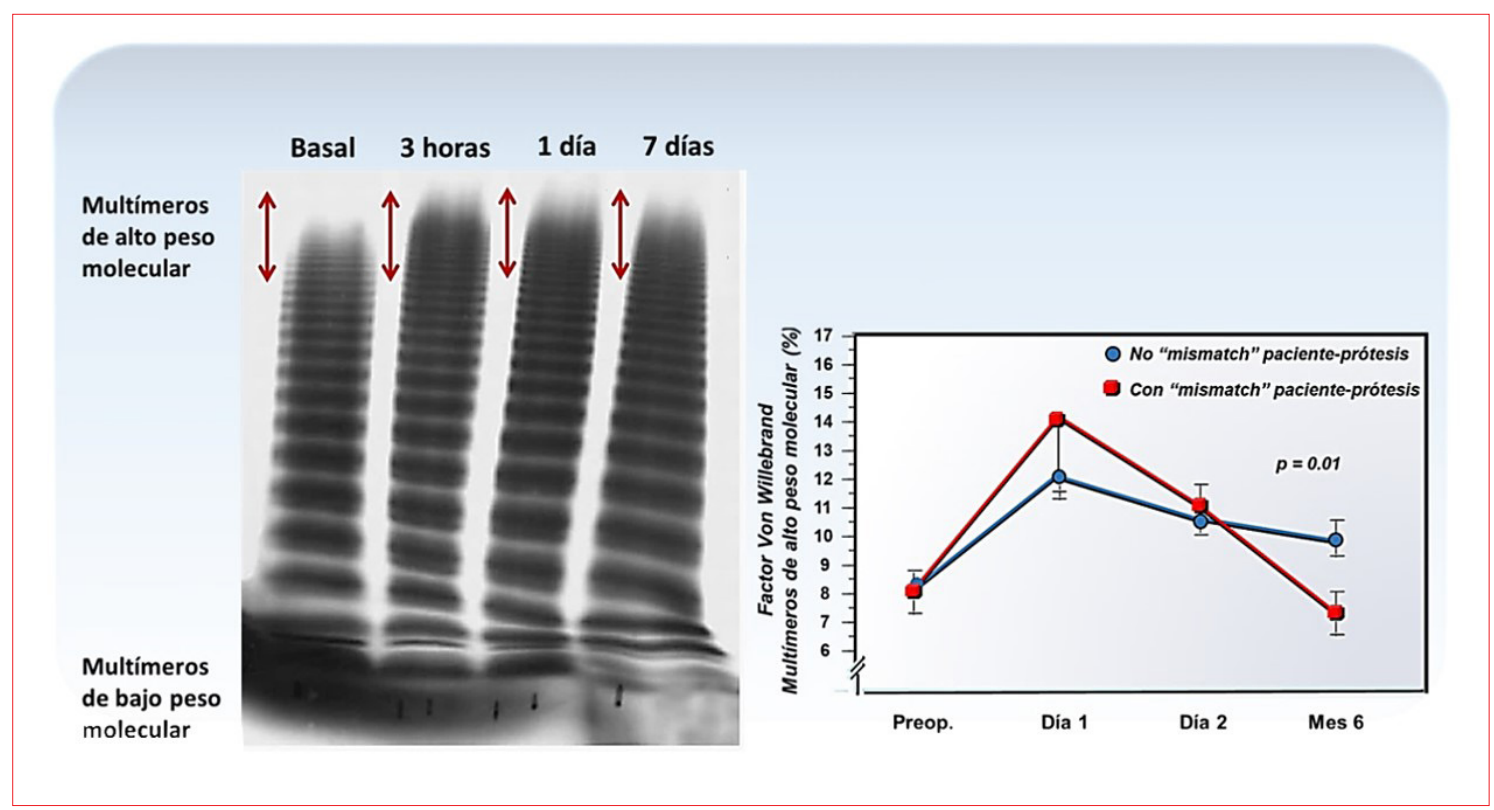

Figura 8. Efecto de la sustitución valvular aórtica en la proporción de los multímeros de alto peso molecular (PM) del factor von Willebrand (fvW). A la izquierda, electroforesis del fvW en gel de agarosa antes y después de la sustitución valvular. Se observa un déficit de los multímeros de alto PM en el preoperatorio y su recuperación luego del procedimiento en varios puntos de corte temporal. Las flechas señalan el área de migración de los multímeros mayores. A la derecha, seguimiento hasta 6 meses del posoperatorio. Luego de su recuperación inicial, las macromoléculas vuelven a caer en forma significativa en los pacientes con mismatch protésico, confirmando la relación causal entre el trastorno hemodinámico propio de la valvulopatía y el defecto de la hemostasis (modificado de Vincentelli y col. $\left.{ }^{(26)}\right)$.

tuvieron un cese prolongado del sangrado, mientras que la sustitución valvular quirúrgica lo logró en el 93\%.

Thompson y col. encontraron que en $79 \%$ de sus pacientes seguidos hasta 15 años el reemplazo valvular resultó curativo para el sangrado (50\% para prótesis mecánica y $85 \%$ para prótesis biológica), y entre los que persistió el sangrado se redujo francamente su frecuencia ${ }^{(39)}$.

Como se mencionó antes, en el estudio de Vincentelli y col.(26) se estableció una correlación inversa entre el gradiente transvalvular y la concentración plasmática de los multímeros de alto PM, demostrando una relación causal entre la estenosis y el defecto hemostático. Más aún: luego de la sustitución valvular comprobaron una recuperación precoz de las macromoléculas del fvW con mejoría funcional plaquetaria en estudios que emulan las condiciones de la hemostasis primaria in vivo, y frecuente recidiva en casos de mismatch prótesis-paciente que reproducen las condiciones de elevadas fuerzas de cizalla de la valvulopatía nativa (figura 8).

Esos resultados versus la resección intestinal de alto riesgo en un paciente con estenosis aórtica severa, probablemente anémico y/o con sangrado activo, determinan que el tratamiento apropiado del síndrome, que resuelve tanto la valvulopatía como su consecuencia - el defecto de la hemostasis y el sangrado- sea la corrección de la lesión valvular. En consecuencia, se ha planteado que, en presencia de estenosis aórtica severa asintomática, la comprobación de un síndrome de Heyde constituiría una indicación formal del procedimiento ${ }^{(40,41)}$, aunque esta conducta aún no ha sido contemplada por las guías de práctica clínica. Esta posición implica que el sangrado o la anemia deberían interpretarse como un síntoma más de la enfermedad valvular.

\section{d) Datos de fisiopatología comparada}

En línea con los datos previos, varias cardiopatías con condiciones hemodinámicas similares a la estenosis aórtica pueden exhibir el mismo impacto sobre la hemostasis. La miocardiopatía hipertrófica (MCH) es un ejemplo destacable.

Si bien desde la década del 70 se han reportado casos aislados de $\mathrm{MCH}$ asociada con sangrado digestivo recurrente por angiodisplasias, Shimizu y col. documentaron por primera vez en 2007 un déficit de los polímeros de alto PM del fvW en un paciente con $\mathrm{MCH}$ obstructiva con elevado gradiente subaórtico y severa anemia por sangrado gastrointestinal oculto ${ }^{(42)}$. El tratamiento con calcioantagonistas, con el correspondiente alivio del gradiente subaórtico, revirtió todas 
las alteraciones. Blackshear y col. publicaron cinco casos de $\mathrm{MCH}$ obstructiva con sangrado gastrointestinal o mucoso y síndrome de $\mathrm{vW}$ adquirido. Todos estos se normalizaron luego de la miectomía septal ${ }^{(43)}$. El mismo resultado se ha informado con la ablación septal con alcohol ${ }^{(44,45)}$. En forma similar a la estenosis aórtica, se ha encontrado una relación cuantitativa independiente entre la caída de los multímeros de alto PM del fvW y el gradiente subaórtico, bastando con $15 \mathrm{mmHg}$ en reposo y $35 \mathrm{mmHg}$ durante ejercicio para afectar su concentración ${ }^{(46)}$. Tal como se ha sugerido, se trataría de una verdadera variante del síndrome de Heyde ${ }^{(47)}$.

Sin embargo, la incidencia del cuadro hemorragíparo es menor en la $\mathrm{MCH}$, probablemente debido al menor promedio de edad de los portadores - con menor prevalencia de angiodisplasiasy a que la obstrucción, si existe, es dinámica y por lo tanto de magnitud variable.

Como ya se ha mencionado, se ha documentado enfermedad de vW adquirida en algunas cardiopatías congénitas con elevados gradientes de presión, como la comunicación interventricular aislada, la tetralogía de Fallot, la estenosis pulmonar y el ductus arterioso permeable ${ }^{(21)}$.

La asistencia circulatoria del ventrículo izquierdo con dispositivos de flujo continuo, en especial de alta velocidad rotacional, con frecuencia es complicada por sangrados por angiodisplasias, inicialmente atribuidos a la anticoagulación requerida por la técnica. Sin embargo, casi todos presentan un déficit selectivo de los multímeros de alto PM del fvW a causa del patrón circulatorio de baja pulsatilidad y elevado shear stress $^{(48)}$.

Las insuficiencias valvulares nativas y las fugas paravalvulares en prótesis implantadas quirúrgicamente o en forma percutánea también pueden generar flujos de alta velocidad y elevados gradientes, ocasionando un patrón hematológico similar $^{(49-51)}$. Esto ha llevado a proponer la valoración funcional del fvW o la cuantificación de sus multímeros de alto PM como nuevos marcadores biológicos de severidad de una valvulopatía nativa o de la eficacia de su corrección ${ }^{(52)}$.

Estas situaciones diversas, con eje fisiopatológico compartido, suman al síndrome de Heyde un definido apoyo como entidad por derecho propio, la cual representa un verdadero nexo entre disciplinas médicas. Su demostración, obtenida con precisión y elegancia, permitió explicar condiciones patológicas muy variadas e introducir un promisorio instrumento de diagnóstico y valoración clínica en el ámbito de la cardiología.

Aunque no es objeto de esta revisión, debe des- tacarse que el origen de un síndrome de vW adquirido no se limita al mecanismo hemodinámico detallado, sino que también puede desencadenarse por procesos físicos o inmunológicos vinculados a síndromes linfo o mieloproliferativos, tumores sólidos, enfermedades autoinmunes, gammapatías monoclonales, enfermedad tiroidea, uremia o acción de fármacos ${ }^{(53)}$.

\section{Cuadro clínico y diagnóstico}

Los pacientes con enfermedad de von Willebrand congénita presentan sangrado excesivo de origen mucocutáneo, consistente en epistaxis recurrentes, gingivorragias, hematomas fáciles, hipermenorrea o sangrado prolongado por laceraciones, en posoperatorios o posparto. La gravedad del sangrado depende del grado del déficit, según se trate del tipo 1 o 3 (tabla 1 ).

En el síndrome de Heyde el cuadro es similar, pero como se trata de un déficit adquirido, aparece en edad más tardía en pacientes con estenosis aórtica conocida o sospechada, sin antecedentes personales ni familiares de sangrado, y con particular participación del tubo digestivo. El sangrado puede ser manifiesto en forma de melenas o enterorragias, con frecuencia severas y/o recurrentes, u oculto, expresándose como una anemia ferropénica con tests positivos para presencia de sangre en materia fecal.

Los estudios endoscópicos convencionales y el laboratorio deben dirigirse a descartar, en primer lugar, patología oncológica o inflamatoria, déficit nutricional y enfermedad celíaca.

Con frecuencia, la exploración digestiva resulta negativa (sangrado de origen oscuro), lo que en un paciente con estenosis aórtica obliga a plantear el síndrome de Heyde como explicación del cuadro. En ausencia de diagnóstico previo de valvulopatía, se impone una atenta auscultación cardíaca en busca de un soplo eyectivo, en especial en el añoso.

El centellograma del pool sanguíneo con glóbulos rojos marcados con ${ }^{99 \mathrm{~m}} \mathrm{Tc}$ tiene baja sensibilidad para la detección de angiodisplasias, y requiere sangrado activo. La arteriografía contrastada mesentérica selectiva es un estudio invasivo no exento de riesgos, y debería involucrar las dos arterias mesentéricas dada la alta prevalencia de angiodisplasias en asas delgadas y en localización multicéntrica.

El advenimiento de la videocápsula endoscópica, con mayor sensibilidad diagnóstica, aunque no siempre disponible, ha mostrado que las angiodisplasias son una causa dominante de hemorra- 


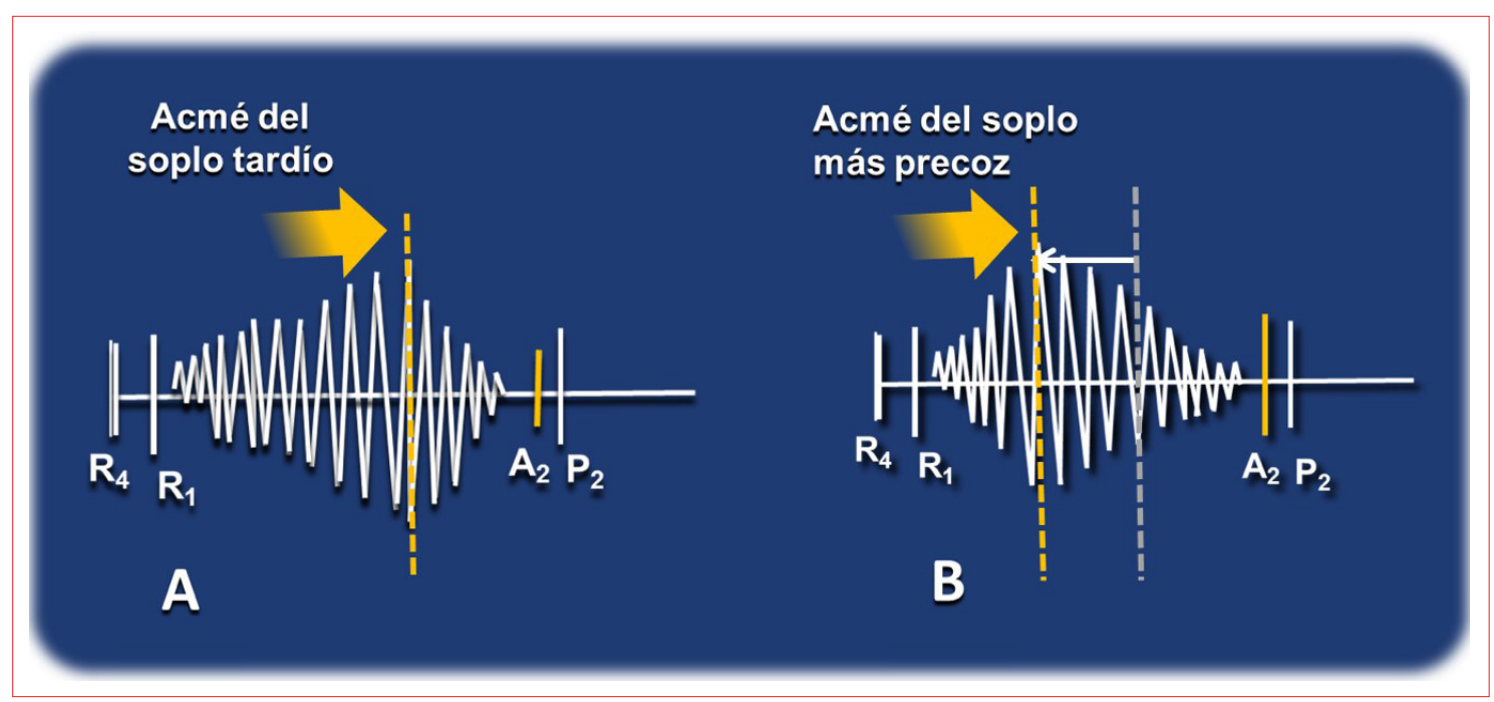

Figura 9. Modificaciones del soplo de estenosis aórtica inducidos por una anemia severa. A: Sin anemia. El soplo característico de la estenosis aórtica severa tiene un acmé tardío, mesotelesistólico. B: Con anemia. El flujo transaórtico instantáneo y, por lo tanto, la intensidad del soplo son máximos en la protomesosístole. La auscultación de un acmé tardío del soplo en un paciente con estenosis aórtica y anemia sugiere severidad de la valvulopatía, si bien la evaluación clínica y ecocardiográfica más confiable tiene lugar luego de su reposición.

gia digestiva en el adulto mayor, en especial del intestino delgado, un segmento mayoritariamente inaccesible a la endoscopía tradicional.

Los estudios básicos de la hemostasis pueden no mostrar alteraciones, e incluso los exámenes específicos para la enfermedad de von Willebrand pueden no resultar categóricos. Debe insistirse en que la enfermedad de vW tipo $2 \mathrm{~A}$ implica un déficit cualitativo, por lo que la concentración del fvW con frecuencia es normal y tiene mayor valor demostrar el deterioro de su actividad biológica (tabla 3).

Los estudios más específicos son la determinación del tiempo de oclusión con el Platelet Function Assay 100 (PFA100), que evalúa la competencia plaquetaria en condiciones de elevado shear stress, y la determinación del porcentaje de multímeros de alto PM del fvW mediante inmunoelectroforesis en gel de agarosa. Sin embargo, su disponibilidad no es universal, y el segundo estudio requiere un procesamiento de al menos una semana, por lo que es un test de confirmación.

El paciente con anemia intensa plantea un problema diagnóstico particular, ya que el síndrome hiperdinámico con aumento de la velocidad circulatoria podría explicar los síntomas e intensificar el soplo eyectivo y el gradiente por Doppler, con lo cual la severidad de la estenosis valvular podría ser sobrevalorada. Por ello, es importante la reconsideración del cuadro y de las decisiones terapéuticas luego de la reposición de glóbulos rojos. No obstante, la auscultación minuciosa del soplo puede orientar hacia el grado de severidad de la valvulopatía (figura 9).
Como contrapartida, puede atribuirse de manera errónea un soplo sistólico exclusivamente a la anemia, en especial si es de intensidad 3/6 o menor y tiene epicentro en el ápex y timbre atípico (soplo de Gallavardin) ${ }^{(54)}$. La investigación del mecanismo y el origen de la anemia, la búsqueda de una discrasia sanguínea y la ecocardiografía orientarán el diagnóstico.

Se ha señalado también que en una estenosis aórtica, aún moderada, puede establecerse un círculo vicioso donde un sangrado con anemia resultante produce incremento en la velocidad circulatoria y del shear stress, mayor déficit del fvW y aumento del sangrado ${ }^{(55)}$.

En suma, el síndrome de Heyde es concebido hoy como una tríada integrada por estenosis aórtica, una diátesis hemorrágica característica y sangrado por angiodisplasias, donde los dos últimos pueden ser de difícil identificación y la valvulopatía de cuantificación incierta. Por lo tanto, es necesario mantener un alto nivel de sospecha en presencia de un soplo eyectivo o una estenosis aórtica conocida asociados a sangrado digestivo o anemia ferropénica no aclarados, en especial en el paciente añoso.

\section{Tratamiento}

Como se ha mencionado, la primera estrategia en el tratamiento del síndrome de Heyde tuvo una finalidad hemostática, procurando corregir un cuadro hemorrágico persistente mediante la resección del segmento intestinal presuntamente 
involucrado en el sangrado(17), en general asiento de angiodisplasias.

Experiencias sucesivas demostraron que, dada la localización incierta o múltiple de las lesiones, muy frecuentemente esta intervención no impedía la recurrencia de las hemorragias, además de introducir el riesgo vital de una cirugía mayor en un paciente con una estenosis aórtica severa y en general sintomática no resuelta.

El tratamiento farmacológico de las angiodisplasias $^{(20,56)}$, una opción disponible desde principios de los años 50, precedió a su vinculación con la estenosis después de la descripción original de Heyde. Los primeros agentes utilizados y quizás los más evaluados fueron los estrógenos y la progesterona, en ocasiones combinados, limitados por contraindicaciones y efectos colaterales. Se sumaron luego varios fármacos con mecanismo de acción variado o no claramente definido, incluyendo el octreótido (un análogo de la somatostatina), corticosteroides, antifibrinolíticos, talidomida, desmopresina y factores VII y VIII de la coagulación. La mayoría de estos fármacos fueron utilizados en sangrados mucocutáneos y su empleo en hemorragia gastrointestinal fue anecdótico o formó parte de pequeñas series, con resultados inconsistentes. Por lo tanto, no hay suficientes estudios controlados ni guías para su uso, que fundamentalmente es profiláctico, como adyuvante de tratamientos locales para reducir el resangrado a largo plazo.

La embolización mediante angiografía selectiva tiene una alta tasa de efectividad, pero no está exenta de complicaciones ocasionalmente severas, exige un equipamiento especial y personal altamente entrenado en radiología vascular. Es más apropiada para un sangrado activo del intestino delgado, dada su inaccesibilidad al tratamiento directo de la lesión.

Los avances tecnológicos permitieron introducir tratamientos endoscópicos de probada eficacia inmediata, aunque con importante recidiva del sangrado. Están indicados en lesiones escasas y restringidas a un sector intestinal accesible al endoscopio. Incluyen la coagulación con argónplasma, la electrocoagulación mono o bipolar y la fotocoagulación mediante distintos tipos de láser, de los cuales los más usados son el Nd:YAG Láser y el Argón Láser. Sin embargo, estas modalidades son de alto costo y requieren gran capacitación técnica ${ }^{(20)}$.

El clipado puede ser una terapia hemostática efectiva en lesiones sangrantes, pero su uso se ha reportado de forma escasa, y preferentemente como tratamiento de rescate.
Con la información disponible, puede afirmase que el tratamiento más apropiado del síndrome de Heyde es la resolución de la obstrucción del tracto de salida del ventrículo izquierdo, que permite tratar sus tres componentes en un solo acto. La sustitución valvular revierte el trastorno fisiopatológico básico, el elevado shear stress del chorro aórtico de alta velocidad, moderando la proteólisis selectiva del fvW por la ADAMTS13 y revirtiendo la consiguiente disfunción plaquetaria y el sangrado de angiodisplasias coincidentes o favorecidas por la propia estenosis aórtica.

La sustitución quirúrgica, única técnica disponible hasta entrado el siglo actual, planteaba el riesgo de un sangrado perioperatorio incontrolable ante un procedimiento invasivo mayor, que incluye entre otras agresiones tisulares una aortotomía y una intensa anticoagulación requerida por la circulación extracorpórea.

También subyacía el temor, ante un sangrado de origen oscuro, que una patología oncológica no diagnosticada condicionara el pronóstico a corto plazo. Es probable que estas consideraciones hayan motivado que la cirugía se descartara en pacientes que por su patología valvular tenían indicación formal. Sin embargo, la experiencia con el uso de la técnica demostró que es segura y efectiva. Se ha observado que el sangrado posquirúrgico de pacientes con alteración preoperatoria de la estructura polimérica del fvW no es significativamente mayor que en los que no la presentan, y que la recuperación de la actividad del fvW y de sus multímeros del alto PM puede producirse tan pronto como en 24 horas $^{(57,58)}$. Esto se debe a una rápida liberación de reservas del fvW desde las células endoteliales y una pronta reversión de su proteólisis acelerada por la enzima ADAMTS13.

Son esenciales la adecuada elección de la oportunidad operatoria y la preparación del paciente, procurando llevarlo al acto sin sangrado activo, sea manifiesto u oculto, y con un aceptable nivel de hemoglobina. Se debe contar con un equipo anestésico-quirúrgico cabalmente informado, con participación de un hemoterapeuta desde el preoperatorio hasta el alta sanatorial, y disponer de hemoderivados y recursos diagnósticos adecuados para la evaluación y la eventual recuperación de la hemostasis (figura 10).

Una condición decisiva para la reversión persistente del defecto hemostático es un implante protésico de diámetro adecuado a la masa corporal de paciente; una prótesis pequeña mantendrá las condiciones de alto shear stress de la valvulopatía nativa, degradación continuada del fvW y recurrencia de sangrados. Otro aspecto 


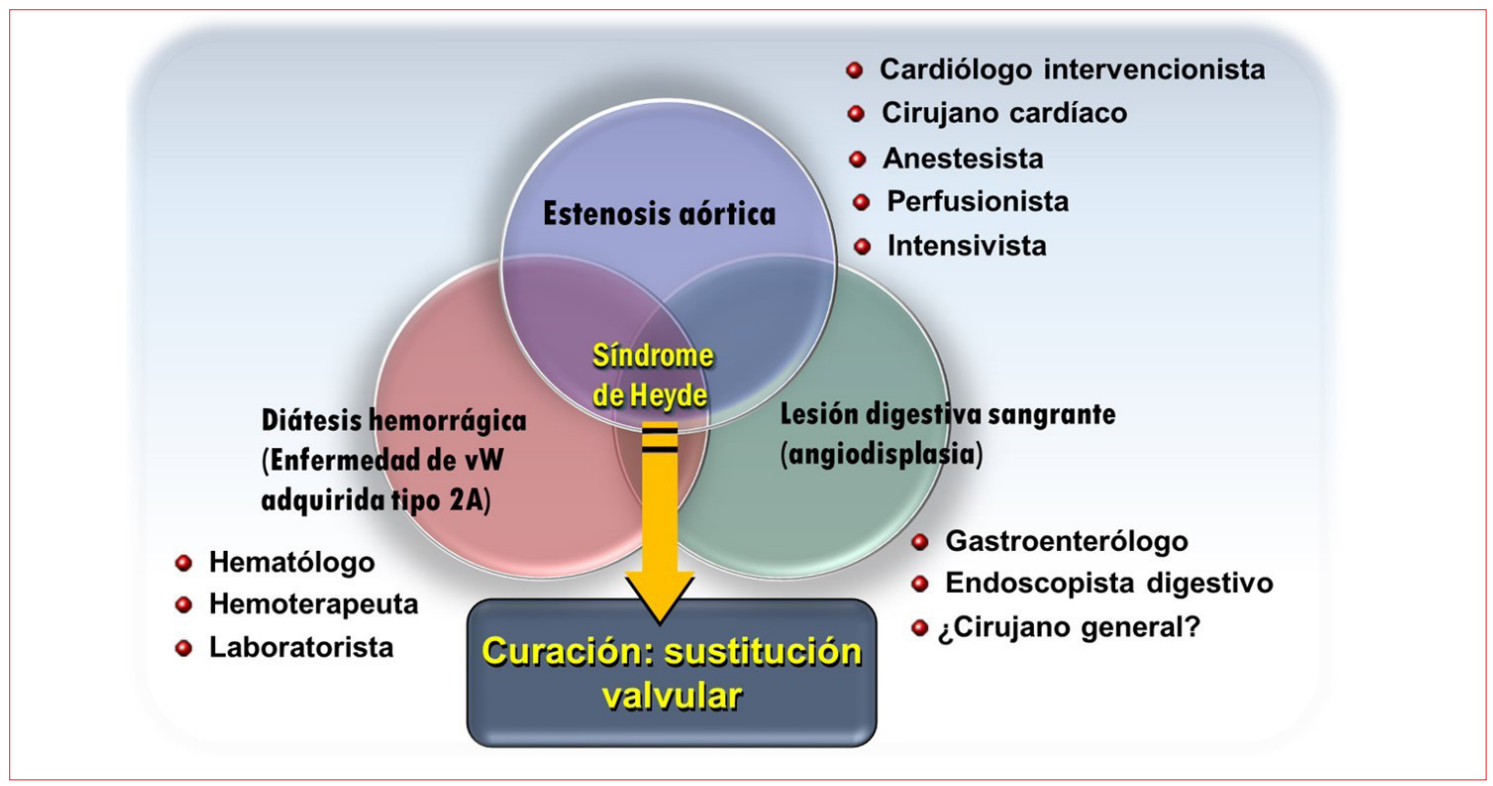

Figura 10. Manejo del síndrome de Heyde: una encrucijada de abordaje multidisciplinario. El síndrome de Heyde es un desorden sistémico integrado por tres componentes con interacciones complejas, cuya comprensión requirió históricamente la participación de varias disciplinas. Del mismo modo, su diagnóstico y su tratamiento, incluyendo elección del procedimiento, oportunidad, preparación y medidas de apoyo ulteriores puede exigir en forma simultánea o sucesiva la colaboración integrada de distintos equipos de especialistas.

Tabla 4. El síndrome de Heyde: su caracterización clínica en 10 puntos.

1. Fue formulado originalmente por Edward Heyde en 1958 como la asociación entre estenosis aórtica severa y hemorragia digestiva grave.

2. Su definición clínico-etiopatogénica y su consolidación como entidad sindromática fue una construcción gradual y colaborativa, con aportes de diferentes disciplinas.

3. Las lesiones sangrantes son habitualmente angiodisplasias submucosas, frecuentes en el colon derecho, pero con posible asiento en cualquier parte del tubo digestivo desde el cardias hasta el esfínter anal, importante participación del intestino delgado y frecuente presencia multifocal.

4. Su presentación se incrementa con la edad, dada la mayor prevalencia de estenosis aórtica y de angiodisplasias con el envejecimiento.

5. Puede manifestarse como un sangrado evidente, con hallazgo de angiodisplasias o sin lesión identificable (sangrado oscuro), o bajo forma de una anemia crónica sin manifestación externa macroscópica (sangrado oculto). Puede acompañarse de sangrados cutáneos o en otras mucosas.

6. El desencadenante del sangrado es una disfunción plaquetaria por déficit de los multímeros de mayor peso molecular del fvW, producto de su proteólisis aumentada por la enzima ADAMTS13. Esta es causada por una alteración conformacional en la que el fvW se despliega desde una forma globular a una fibrilar, lo que aumenta la exposición de su punto de escisión. El origen de este cambio está en las elevadas fuerzas de cizalla a nivel del chorro de alta velocidad de la estenosis valvular. Los multímeros en déficit son particularmente necesarios para la hemostasis en vasos de alto flujo como las angiodisplasias.

7. Una vez dilucidada su fisiopatología, el síndrome queda configurado como la tríada de estenosis aórtica, déficit cualitativo del $\mathrm{fvW}$ — catalogado como una enfermedad de von Willebrand adquirida de tipo $2 \mathrm{~A}$ - y sangrado digestivo o su consecuencia, la anemia ferropénica crónica.

8. El tratamiento adecuado es la sustitución valvular, que resuelve en la mayoría de los casos tanto la estenosis como la tendencia al sangrado y el trastorno hemostático de base, y es más efectiva y menos riesgosa que la laparotomía con resección intestinal.

9. En principio, la TAVR es preferible a la sustitución quirúrgica, dada su menor invasividad en pacientes generalmente añosos, anémicos y con comorbilidades, y una adecuada área efectiva de la prótesis. Sin embargo, es esencial evitar una regurgitación paravalvular significativa.

10. Su diagnóstico requiere un elevado índice de sospecha, descartando malignidad -y también enfermedad celíaca y déficit nutricional en caso de una anemia crónica- y su tratamiento implica la participación informada e interactiva de diversas especialidades que incluyen la cardiología clínica, la hematología, la gastroenterología, el laboratorio y la cardiología invasiva/cirugía cardíaca. 
Tabla 5. Importancia del reconocimiento clínico del síndrome de Heyde.

Permite identificar, en un paciente portador de estenosis aórtica, el mecanismo de un sangrado gastrointestinal manifiesto o de un síndrome anémico crónico sin causa determinable con los estudios digestivos.

Orienta hacia el tratamiento adecuado de la valvulopatía y del cuadro hemorragíparo: el reemplazo valvular aórtico, frecuentemente descartado por temor al sangrado masivo periprocedimiento.

Influye en la discusión sobre el tipo de prótesis a implantar (biológica versus mecánica), dada la posible incidencia de un tratamiento anticoagulante a largo plazo sobre la recurrencia del sangrado.

Incide en la elección de la técnica de sustitución valvular (SAVR o TAVR), su oportunidad y la consideración de posibles medidas de apoyo hemostático y hematológico periprocedimiento.

Refuerza la necesidad de un sustituto valvular de buen comportamiento hemodinámico, para evitar la persistencia o recidiva de la diátesis hemorrágica.

Plantea el posible papel que pudiera tener esta asociación en la indicación per se del tratamiento intervencionista en la estenosis aórtica asintomática en futuras revisiones de las guías.

El conocimiento de su fisiopatología ha permitido considerar al fvW como un biomarcador, tanto de la severidad de una lesión valvular nativa como de la eficacia hemodinámica de un implante protésico o de una reparación valvular.

SAVR: sustitución quirúrgica de válvula aórtica; TAVR: implante percutáneo de válvula aórtica.

para considerar en forma individualizada es la elección del tipo de prótesis. Si bien la respuesta precoz del defecto hemostático en portadores de sustitutos mecánicos es adecuada, la recidiva de sangrados en pacientes anticoagulados parece ser mayor que entre quienes reciben prótesis biológicas ${ }^{(39)}$.

Desde $2013^{(59)}$ se han publicado varios reportes sobre implante valvular aórtico percutáneo (TAVR) en pacientes con síndrome de Heyde. La TAVR se presenta como una opción atractiva en este contexto, por su menor invasividad en un paciente frecuentemente añoso y con comorbilidades y porque evita la incisión aórtica y el uso de circulación extracorpórea con su correspondiente heparinización de alta intensidad. Además, por lo común obtiene un área protésica efectiva algo mayor que una prótesis biológica quirúrgica. El comportamiento luego del implante es en general comparable al observado con la sustitución quirúrgica, con resolución de la anomalía hemostática de fondo y los sangrados. En cuanto a comparaciones directas a gran escala, solo encontramos un registro en el que no hubo diferencias en mortalidad, pero la TAVR mostró menor tasa de stroke, transfusiones y tiempo de internación ${ }^{(60)}$.

Sin embargo, también se reportan sangrados periprocedimento ${ }^{(59)}$, recuperación tardía(61) y reaparición diferida del cuadro ${ }^{(62)}$. Es probable que ello esté vinculado a que en un porcentaje no menor de los procedimientos se registra una regurgitación periprotésica significativa y de alta velocidad, que mantiene condiciones de shear stress elevado, con degradación persistente del fvW ${ }^{(63)}$. Es reconocido que un gradiente residual o una regurgitación mayor que leves son predictores de evolución a mediano y largo plazo. Van Belle y col. demostraron una estrecha correlación entre regurgitación periprotésica pos-TAVR (14\% de sus pacientes), persistencia de bajo nivel de multímeros de alto PM del fvW, disfunción plaquetaria no corregida y mortalidad a un año del procedimiento ${ }^{(64)}$. El mismo grupo comprobó que los cambios en la función plaquetaria evaluada con el PFA100 reflejan de manera fiel los del patrón molecular del fvW, y ocurren escasos minutos luego de las modificaciones de flujo y shear stress introducidas por el implante. Esta respuesta tan dinámica permitiría disponer de un medio de evaluación del resultado del implante en tiempo real adicional al ultrasonido ${ }^{(65)}$.

Un aspecto aún no definido es el tratamiento antitrombótico ideal en estos pacientes, considerando el posible efecto de la antiagregación dual sobre el riesgo de sangrado pos-TAVR. Se ha informado que el uso de tromboelastometría rotacional podría servir de referencia para definir la necesidad de transfusión plaquetaria y el momento del inicio del tratamiento antiagregante ${ }^{(66)}$.

En suma, el patrón de oro en el tratamiento del síndrome de Heyde es la sustitución valvular, preferentemente mediante implante percutáneo, a condición de obtener un resultado hemodinámico plenamente satisfactorio, objetivo para cuyo control intraprocedimiento se perfila un nuevo y promisorio método.

En la tabla 4 se sintetiza el perfil clínico-fisiopatológico del síndrome de Heyde en 10 conceptos esenciales. 


\section{Conclusiones}

Han transcurrido más de sesenta años desde la perspicaz observación de Heyde, realizada en un contexto en que el diagnóstico de la estenosis aórtica era exclusivamente clínico, no admitía tratamiento específico alguno y la endoscopía colónica aún no se había desarrollado.

Los sucesivos avances en diversos campos de la medicina confirmaron su realidad, y el esclarecimiento de sus mecanismos íntimos tuvo derivaciones entonces insospechadas en la comprensión y manejo, tanto de la estenosis aórtica como de entidades fisiopatológicamente emparentadas.

La necesidad del conocimiento profundo sobre este síndrome trasciende, por lo tanto, a la mera confirmación de un planteo original y al justo reconocimiento a su autor; es condición indispensable para la detección e interpretación adecuada de sus manifestaciones y el manejo apropiado de la valvulopatía, a resolver necesariamente en un entorno multidisciplinario.

Las conclusiones más relevantes, que remiten entonces a enfatizar el valor práctico de su reconocimiento, se esquematizan en la tabla 5 .

\section{Jorge Estigarribia Passaro,}

ORCID: 0000-0000-5136-7668

Este artículo fue aceptado para su publicación por el Editor jefe Dr. Federico Ferrando

\section{Bibliografía}

1. Heyde EC. Gastrointestinal bleeding in aortic stenosis. New Engl J Med. 1958; 259: 196. doi: 10.1056/ NEJM195807242590416.

2. Schwartz BM. Additional note on bleeding in aortic stenosis. New Engl J Med. 1958; 259: 456. doi: 10.1056/NEJM195808282590918.

3. Goldman MJ. Aortic stenosis and gastrointestinal bleeding. New Engl J Med. 1958; 259: 941. doi: 10.1056/NEJM195811062591921

4. Apostolakis E, Doering C, Kantartzis M, Winter J, Shulte HD. Calcific aortic-valve stenosis and angiodysplasia of the colon: Heyde's syndrome. Report of two cases. Thorac Cardiovasc Surg. 1990; 38 (6): 3746. doi: 10.1055/s-2007-1014055.

5. Boley SJ, Brandt LJ. Vascular ectasias of the colon-1986. Dig Dis Sci. 1986; 31 (9): 26S42S. doi: 10.1007/BF01295988.

6. Williams RC Jr. Aortic stenosis and unexplained gastrointestinal bleeding. Arch Intern Med. 1961; 108: 85963. doi: 10.1001/archinte.1961. 03620120043007 .

7. McNamara JJ, Austen WG. Gastrointestinal bleeding occurring in patients with acquired valvular disease. Arch Surg. 1968; 97 (4): 53840. doi: 10.1001/archsurg.1968.01340040034003.

8. Cody MC, O'Donovan TP, Hughes RW Jr. Idiopathic gastrointestinal bleeding and aortic stenosis. Am J Dig Dis. 1974; 19 (5): 3938. doi: 10.1007/ BF01255601.

9. Shoenfeld Y, Eldar M, Bedazovsky B, Levy MJ, Pinkhas J. Aortic stenosis associated with gastrointestinal bleeding. A survey of 612 patients. Am Heart J. 1980; 100 (2): 179-82. doi: 10.1016/00028703(80)90113-1.

10. Greenstein RJ, McElhinney AJ, Reuben D, Greenstein AJ. Colonic vascular ectasias and aortic stenosis: coincidence or causal relationship? Am J Surg. 1986; 151 (3): 34751. doi: 10.1016/00029610(86)90465-4.

11. Imperiale TF, Ransohoff DF. Aortic stenosis, idiopathic gastrointestinal bleeding, and angiodysplasia. Is there an association? A methodologic critique of the literature. Gastroenterology. 1988; 95 (6): 16706. doi: 10.1016/S0016-5085(88)80095-7.

12. Mehta PM, Heinsimer JA, Bryg RJ, Jaszewski R, Wynne J. Reassessment of the association between gastrointestinal arteriovenous malformations and aortic stenosis. Am J Med. 1989; 86 (3): 2757. doi: 10.1016/0002-9343(89)90295-7.

13. Oneglia C, Sabatini T, Rusconi C, Gardini A, Paterlini A, Buffoli $\mathbf{F}$ et al. Prevalence of aortic valve stenosis in patients affected by gastrointestinal angiodysplasia. Eur J Med. 1993; 2 (2): 758. PMID: 8258021.

14. Bhutani MS, Gupta SC, Markert RJ, Barde CJ, Donese R, Gopalswamy N. A prospective controlled evaluation of endoscopic detection of angiodysplasia and its association with aortic valve disease. Gastrointest Endoscop. 1995; 42 (5): 398402. doi: 10.1016/s0016-5107(95)70038-2.

15. Batur P, Stewart WJ, Isaacson H. Increased prevalence of aortic stenosis in patients with arteriovenous malformations of the gastrointestinal tract in Heyde syndrome. Arch Intern Med. 2003; 163 (15): 18214. doi: 10.1001/archinte.163.15.1821.

16. Pate GE, Mulligan A. An epidemiological study f Heyde's syndrome: an association between aortic stenosis and gastrointestinal bleeding. J Heart Valve Dis. 2004; 13 (5): 7136. PMID: 15473467.

17. Castleman B, McNeely BU. Case records of the Massachusetts General Hospital. Weekly clinicopathological exercises. Case 49-1965. N Engl J Med. 1965; 273 (20): 1096105. doi: 10.1056/ NEJM196511112732010.

18. Boss EG Jr, Rosenbaum JM. Bleeding from the right colon associated with aortic stenosis. Am J Dig Dis. 1971; 16 (3): 26975. doi: 10.1007/BF02235250.

19. Shbeeb I, Prager E, Love J. The aortic valve. Colonic axis. Dis Colon Rectum. 1984; 27 (1): 3841. doi: 10.1007/BF02554072.

20. García-Compeán D, Del Cueto-Aguilera ÁN, Jiménez-Rodríguez AR, González-González JA, Maldonado-Garza HJ. Diagnostic and therapeutic 
challenges of gastrointestinal angiodysplasias: a critical review and view points. World J Gastroenterol. 2019; 25 (21): 254964. doi: 10.3748/wjg.v25.i21.2549.

21. Gill JC, Wilson AD, Endres-Brooks J, Montgomery RR. Loss of the largest von Willebrand factor multimers from the plasma of patients with congenital cardiac defects. Blood. 1986; 67 (3): 75861. doi: 10.1182/blood.V67.3.758.bloodjournal673758.

22. Warkentin TE, Moore JC, Morgan DG. Aortic stenosis and bleeding gastrointestinal angiodysplasia: is acquired von Willebrand's disease the link? Lancet 1992; 340 (8810): 357. doi: 10.1016/01406736(92)92434-h.

23. Tsai HM, Sussman II, Nagel RL. Shear stress enhances the proteolysis of von Willebrand factor in normal plasma. Blood. 1994; 83 (8): 21719. doi: 10.1182/blood.V83.8.2171.2171.

24. Pareti FI, Lattuada A, Bressi C, Zanobini M, Sala A, Steffan A et al. Proteolysis of von Willebrand factor and shear stress-induced platelet aggregation in patients with aortic valve stenosis. Circulation. 2000; 102 (11): 12905. doi: 10.1161/01. cir.102.11.1290

25. Fujikawa K, Suzuki H, McMullen B, Chung D. Purification of human von Willebrand factor-cleaving protease and its identification as a new member of the metalloproteinase family. Blood. 2001; 98 (6): 16626. doi: 10.1182/blood.v98.6.1662.

26. Vincentelli A, Susen S, Le Tourneau T, Six I, Fabre O, Juthier $\mathbf{F}$ et al. Acquired von Willebrand syndrome in aortic stenosis. N Engl J Med. 2003; 349 (4): 3439. doi: 10.1056/NEJMoa022831.

27. Bonow RO, Carabello BA, Chatterjee K, De León AC Jr, Faxon DP, Freed MD et al. 2008 focused update incorporated into the ACC/AHA 2006 Guidelines for the management of patients with valvular heart disease: A report of the American College of Cardiology/American Heart Association Task Force on Practice Guidelines (Writing Committee to revise the 1998 guidelines for the management of patients with valvular heart disease). Circulation. 2008; 118 (15): e523e661. doi: 10.1161/ CIRCULATIONAHA.108.190748.

28. Lenting PJ, Pegon JN, Groot E, De Groot PG. Regulation of von Willebrand-platelet interactions. Thromb Haemost. 2010; 104 (3): 44955. doi: 10.1160/TH09-11-0777.

29. Crawley JTB, De Groot R, Xiang Y, Luken BM, Lane DA. Unraveling the scissile bond: how ADAMTS13 recognizes and cleaves von Willebrand factor. Blood. 2011; 118 (12): 321221. doi: 10.1182/ blood-2011-02-306597.

30. Mehta R, Athar M, Girgis S, Hassan A, Becker RC. Acquired von Willebrand syndrome (AVWS) in cardiovascular disease: a state of the art review for clinicians. J Thromb Thrombolysis. 2019; 48 (1): 1426. doi: 10.1007/s11239-019-01849-2.

31. Rauch A, Wohner N, Cristophe OD, Denis CV, Susen S, Lenting PJ. On the versatility of von Willebrand factor. Mediterr J Hematol Infect Dis. 2013; 5 (1): e2013046. doi: 10.4084/MJHID.2013.046.
32. Starke RD, Ferraro F, Paschalaki KE, Dryden NH, McKinnon TAJ, Sutton RE et al. Endothelial von Willebrand factor regulates angiogenesis. Blood. 2011; 117 (3): 107180. doi: 10.1182/ blood-2010-01-264507.

33. Randi AM, Smith KE, Castaman G. Von Willebrand factor regulation of blood vessel formation. Blood. 2018; 132 (2): 13240. doi: 10.1182/ blood-2018-01-769018.

34. Cappell MS, Lebwohl O. Cessation of recurrent bleeding from gastrointestinal angiodysplasias after aortic valve replacement. Ann Intern Med. 1986; 105 (1): 547. doi: 10.7326/0003-4819-105-1-54

35. Tsuchiya S, Matsumoto Y, Doman T, Fujiya T, Sugisawa J, Suda A et al. Dissapearance of angiodysplasia following transcatheter aortic valve implantation in a patient with Heyde's syndrome. A case report and review of the literature. J Atheroscler Thromb. 2020; 27 (3): 2717. doi: 10.5551/ jat.49239.

36. Sami SS, Al-Araji SA, Ragunath K. Review article: gastrointestinal angiodysplasia-pathogenesis, diagnostic and management. Aliment Pharmacol Ther. 2014; 39 (1): 1534. doi: 10.1111/apt.12527.

37. Sucker C. The Heyde syndrome: proposal for an unifying concept explaining the association of aortic valve stenosis, gastrointestinal angiodysplasia and bleeding. Int J Cardiol. 2007; 115 (1): 778. doi: 10.1016/j.ijcard.2006.01.014.

38. King RM, Pluth JR, Giuliani ER. The association of unexplained gastrointestinal bleeding with calcific aortic stenosis. Ann Thorac Surg. 1987; 44 (5): 5146. doi: 10.1016/S0003-4975 (10) 62112-1.

39. Thompson JL III, Schaff HV, Dearani JA, Park SJ, Sundt TM III, Suri RM et al. Risk of recurrent gastrointestinal bleeding after aortic valve replacement in patients with Heyde syndrome. J Thorac Cardiovasc Surg. 2012; 144 (1): 1126. doi: 10.1016/j. jtcvs.2011.05.034.

40. Leibovitz E, Harpaz D, Elly I, Klepfish A, Gavish D. Persistent anemia in otherwise asymptomatic severe aortic stenosis. a possible indication for valve replacement? Isr Med Assoc J. 2004; 6 (7): 4002. PMID: 15274529.

41. Michot J-M, Treton X, Brink C, Fabiani J-N, Bouhnik Y. Severe gastro-intestinal angiodysplasia in context of Heyde's syndrome durably cured after aortic valve replacement. Presse Med. 2012; 41 (7-8): 7636. doi: 10.1016/j.lpm.2011.10.012.

42. Shimizu M, Masai H, Miwa Y. Occult gastrointestinal bleeding due to acquired von Willebrand syndrome in a patient with hypertrophic obstructive cardiomyopathy. Intern Med. 2007; 46 (8): 4815. doi: 10.2169/internalmedicine.46.6026.

43. Blackshear JL, Shaff HV, Ommen SR, Chen D, Nichols WL. Hypertrophic obstructive cardiomyopathy, bleeding history, and acquired von Willebrand syndrome: response to septal myectomy. Mayo Clin Proc. 2011; 86 (3): 21924. doi: 10.4065/ mcp.2010.0309.

44. Riis Hansen P, Hassager C. Septal alcohol ab- 
lation and Heyde's syndrome revisited. J Intern Med. 2003; 253 (4): 4901. doi: 10.1046/j.13652796.2003.01127.x.

45. Hvid-Jensen HS, Poulsen SH, Agnholt JS. Severe gastrointestinal bleeding in a patient with subvalvular aortic stenosis treated with thalidomide and octreotide: bridging to transcoronary ablation of septal hypertrophy. J Clin Med Res. 2015; 7 (11): 90710. doi: 10.14740/jocmr2321w.

46. Le Tourneau T, Susen S, Caron C, Millaire A, Maréchaux S, Polge A-S et al. Functional impairment of von Willebrand factor in hypertrophic cardiomyopathy: relation to rest and exercise obstruction. Circulation. 2008; 118 (15): 15507. doi: 10.1161/CIRCULATIONAHA.108.786681.

47. Martí L, Antón R, Almela P, Benages A. Angiodisplasia intestinal asociada a estenosis subaórtica hipertrófica: ¿una variante del síndrome de Heyde? Med Clin (Barc). 2005; 125 (16): 6359. doi: $10.1157 / 13080832$.

48. Blackshear JL. Heyde syndrome. aortic stenosis and beyond. Clin Geriatr Med. 2019; 35 (3): 36979. doi: 10.1016/j.cger.2019.03.007.

49. Blackshear JL, Wysokinska EM, Safford RE, Thomas CS, Shapiro BP, Ung S et al. Shear stress-associated acquired von Willebrand syndrome in patients with mitral regurgitation. J Thromb Haemost. 2014; 12 (12): 196674. doi: $10.1111 /$ jth.12734.

50. Kasai M, Osako M, Inaba Y, Yamabe K, Aoki M. Acquired von Willebrand syndrome secondary to mitral and aortic regurgitation. J Card Surg. 2020; 35 (9): 23968. doi: 10.1111/jocs.14796.

51. Blackshear JL, McRee CW, Safford RE, Pollak PM, Stark ME, Thomas CS et al. Von Willebrand factor abnormalities and Heyde syndrome in dysfunctional heart valve prostheses. JAMA Cardiol. 2016; 1 (2): 198204. doi: 10.1001/jamacardio.2016.0075.

52. Blackshear JL, Wysokinska EM, Stafford RE, Thomas CS, Stark ME, Shapiro BP et al. Indexes of von Willebrand factor as biomarkers of aortic stenosis severity (from the Biomarkers of Aortic Stenosis Severity [BASS] Study). Am J Cardiol. 2013; 111 (3): 37481. doi: 10.1016/j.amjcard.2012.10.015.

53. Federici AB, Rand JH, Bucciarelli P, Budde U, van Genderen PJJ, Mohri $\mathbf{H}$ et al. Acquired von Willebrand syndrome: data from an international registry. On behalf of the Subcommittee on von Willebrand Factor. Thromb Haemost. 2000; 84 (2): 3459. doi: 10.1055/s-0037-1614018.

54. Jolobe OMP. Other aspects of Heyde's syndrome. Am J Emerg Med. 2021; 46: 674. doi: 10.1016/j. ajem.2020.08.027.

55. Schwaiger JP, Ludwicsek O, Graziadei I, Grander W. A vicious circle: Heyde syndrome in mild aortic stenosis. CASE (Phila). 2019; 3 (4): 1716. doi: 10.1016/j.case.2019.04.005.

56. Szilagyi A, Ghali MP. Pharmacological therapy of vascular malformations of the gastrointestinal tract. Can J Gastroenterol. 2006; 20 (3): 1718. doi: 10.1155/2006/859435.

57. Bolliger D, Dell-Kuster S, Seeberger MD, Tanaka KA, Gregor M, Zenklusen U et al. Impact of loss of high-molecular-weight von Willebrand factor multimers on blood loss after aortic valve replacement. Br J Anaesth. 2012; 108 (5): 75462. doi: 10.1093/bja/aer512.

58. Yamashita K, Yagi H, Hayakawa M, Abe T, Hayata Y, Yamaguchi N et al. Rapid restoration of thrombus formation and high-molecular-weight von Willebrand factor multimers in patients with severe aortic stenosis after valve replacement. J Atheroscler Thromb. 2016; 23 (10): 11508. doi: 10.5551/ jat.34421.

59. Godino C, Lauretta L, Pavon AG, Mangieri A, Viani G, Chieffo A et al. Heyde's syndrome incidence and outcome in patients undergoing transcatheter aortic valve implantation. J Am Coll Cardiol. 2013; 61 (6): 6879. doi: 10.1016/j. jacc.2012.10.041.

60. Desai R, Gupta S, Singh S, Bhuva R, Zalavadia D, Parekh T et al. Outcomes of SAVR versus TAVR in aortic stenosis hospitalizations with angiodysplasia-associated gastrointestinal bleeding (Heyde's syndrome): insights from a nationwide propensity-matched analysis [internet]. J Am Coll Cardiol. 2019 [consultado el 22 de mayo de 2021]; 73 (9): 1240. Disponible en: https://www.jacc.org/ doi/abs/10.1016/S0735-1097\%2819\%2931847-9.

61. CP Balbo, Seabra LP, Galoro VG, Caputi G, Palma JH, Buffolo Ê. Heyde's syndrome and transcatheter aortic valve implantation. Arq Bras Cardiol. 2017; 108 (4): 37880. doi: 10.5935/ abc. 20160193 .

62. Alshuwaykh O, Krier MJ. A case of Heyde syndrome with resolution of gastrointestinal bleeding two weeks after aortic valve replacement. Am J Case Rep. 2018; 19: 924-6. doi: 10.12659/AJCR.911298.

63. Spangenberg T, Budde U, Schewel D, Frerker C, Thielsen T, Kuck K-H et al. Treatment of acquired von Willebrand syndrome in aortic stenosis with transcatheter aortic valve replacement. JACC Cardiovasc Interv. 2015; 8 (5): 692700. doi: 10.1016/j.jcin.2015.02.008.

64. Van Belle E, Rauch A, Vincent F, Robin E, Kibler M, Labreuche $\boldsymbol{J}$ et al. Von Willebrand factor multimers during transcatheter aortic-valve replacement. N Engl J Med. 2016; 375: 33544. doi: 10.1056/NEJMoa1505643.

65. Van Belle E, Rauch A, Vincentelli A, Jeanpierre E, Legendre P, Juthier $\mathbf{F}$ et al. Von Willebrand factor as a biological sensor of blood flow to monitor percutaneous aortic valve interventions. Circ Res. 2015; 116 (7): 1193201. doi: 10.1161/CIRCRESAHA.116.305046.

66. Fukuhara K, Kondo T, Miyoshi H, Hamada H, Kawamoto M. Rotational thromboelastometry-guided perioperative management of coagulation in a patient with Heyde's syndrome undergoing transcatheter aortic valve implantation. JA Clin Rep. 2019; 5 (1):3. doi: 10.1186/s40981-019-0224-3. 
67. Nichols WL (Chair), Horton A, Raphael M, Creech C, Scalia E, Banks H et al. The diagnosis, evaluation and management of von Willebrand disease. Bethesda: National Heart Lung and Blood Institute. U.S. Department of Health and Human Services. National Institutes of Health; 2007.

68. Veyradier A, Balian A, Wolf M, Giraud V, Montembault S, Obert B et al. Abnormal von Willebrand factor in bleeding angiodysplasias of the digestive tract. Gastroenterology. 2001; 120 (2):
34653. doi: 10.1053/gast.2001.21204.

69. Warkentin TE, Moore JC, Morgan DG. Gastrointestinal angiodysplasia and aortic stenosis. N Engl J Med. 2002; 347 (11): 8589. doi: 10.1056/ NEJM200209123471122.

70. Yoshida K, Tobe S, Kawata M, Yamaguchi M. Acquired and reversible von Willebrand disease with high shear stress aortic valve stenosis. Ann Thorac Surg. 2006; 81 (2): 4904. doi: 10.1016/j.athoracsur.2005.07.074.

El autor elaboró en su totalidad el presente artículo. 